\title{
Dynamic Flow Distortion Investigation in an S-duct using DDES and SPIV data
}

\author{
Daniel Gil-Prieto ${ }^{1}$, David G. MacManus² (MAIAA), Pavlos K. Zachos ${ }^{3}$, Geoffrey Tanguy ${ }^{4}$, François Wilson ${ }^{5}$, \\ Nicola Chiereghin ${ }^{6}$ \\ Cranfield University, Cranfield, England, MK43 0AL, United Kingdom
}

The dynamic flow distortion generated within convoluted aero-engine intakes can affect the performance and operability of the engine. There is a need for a better understanding of the main flow mechanisms which promote flow distortion at the exit of S-shaped intakes. This paper presents a detailed analysis of the main coherent structures in an S-duct flow field based on a Delayed Detached Eddy Simulation. The capability of this numerical approach to capture the characteristics of the highly unsteady flow field is demonstrated against high resolution, synchronous Stereoscopic Particle Image Velocimetry measurements at the Aerodynamic Interface Plane. The flow field mechanisms responsible for the main perturbations at the duct outlet are identified. Clockwise and counter-clockwise stream-wise vortices are alternately generated around the separation region at a frequency of $\mathrm{St}=\mathbf{0 . 5 3}$, which promote the swirl switching at the duct outlet. Spanwise vortices are also shed from the separation region at a frequency of $\mathrm{St}=1.06$, and convect downstream along the separated centreline shear layer. This results in a vertical modulation of the main loss region and a fluctuation of the velocity gradient between the high and low velocity flow at the Aerodynamic Interface Plane.

\section{Nomenclature}

$a$

$A R$

$D$
$=$ POD temporal coefficient, $\mathrm{m} / \mathrm{s}$

$=$ Area Ratio

$=\mathrm{S}$-duct cross section diameter, $\mathrm{mm}$

\footnotetext{
${ }^{1}$ PhD Student, Propulsion Engineering Centre, Building 52, Cranfield University.

2 Senior Lecturer, Propulsion Engineering Centre, Building 52, Cranfield University, AIAA Member.

${ }^{3}$ Lecturer, Propulsion Engineering Centre, Building 52, Cranfield University, AIAA Member.

${ }^{4} \mathrm{PhD}$ Student, Propulsion Engineering Centre, Building 52, Cranfield University.

${ }^{5}$ Researcher, Propulsion Engineering Centre, Building 52, Cranfield University.

${ }^{6}$ Researcher, Propulsion Engineering Centre, Building 52, Cranfield University.
} 


\begin{tabular}{|c|c|c|}
\hline$H$ & $=$ & S-duct centerline offset, $\mathrm{mm}$ \\
\hline$L$ & $=$ & S-duct axial length, mm \\
\hline$L_{s}$ & $=$ & S-duct length measured along the centerline, $\mathrm{mm}$ \\
\hline$K E$ & $=$ & Kinetic Energy, $\mathrm{J} / \mathrm{kg}$ \\
\hline$M$ & $=$ & Mach number \\
\hline$R$ & $=$ & S-duct cross section radius, $\mathrm{mm}$ \\
\hline$R_{c}$ & $=$ & Curvature radius of the $\mathrm{S}$-duct bend, $\mathrm{mm}$ \\
\hline $\operatorname{Re}_{D}$ & $=$ & Reynolds number based on the inlet diameter \\
\hline$s$ & $=$ & S-duct centerline co-ordinate, $\mathrm{mm}$ \\
\hline$S I$ & $=$ & Swirl Intensity distortion descriptor, deg \\
\hline$S t$ & $=$ & Strouhal number, $f D_{A I P} /\left\langle\bar{w}_{A I P}\right\rangle$ \\
\hline$t_{c}$ & $=$ & S-duct convective time, $L_{s} / w_{\text {in }}, \mathrm{s}$ \\
\hline$T K E$ & $=$ & Turbulent Kinetic Energy, J/kg \\
\hline$u, v, w$ & $=$ & Velocity vector cartesian components, $\mathrm{m} / \mathrm{s}$ \\
\hline$V_{i p}$ & $=$ & In-plane velocity modulus, $\sqrt{u^{2}+v^{2}}, \mathrm{~m} / \mathrm{s}$ \\
\hline$v_{\theta}$ & $=$ & Circumferential velocity component, $\mathrm{m} / \mathrm{s}$ \\
\hline$\alpha$ & $=$ & Swirl angle, $\arctan \left(v_{\theta} / w\right), \operatorname{deg}$ \\
\hline$\gamma$ & $=$ & Curvature ratio based on the inlet-section radius, $R_{\text {in }} / R_{c}$ \\
\hline$\Delta t$ & $=$ & DDES simulation time step, $\mathrm{s}$ \\
\hline$\Phi$ & $=$ & POD non-dimensional modal distribution \\
\hline
\end{tabular}

\section{Subscripts}

AIP $=$ Aerodynamic Interface Plane $\left(0.24 \mathrm{D}_{\text {out }}\right.$ downstream of the S-duct outlet plane $)$

FSM $\quad=$ First Switching Mode

FVM $\quad=$ First Vertical Mode

in $\quad=$ S-duct inlet plane

$\max \quad=$ Maximum value of a temporal distribution

mean $=$ Time-averaged value of a temporal distribution 


$\begin{array}{ll}\text { MFM } & =\text { Mean-Flow Mode } \\ \text { out } & =\text { S-duct outlet plane } \\ r e f & =\text { Reference plane }\left(0.9 \mathrm{D}_{\text {in }} \text { upstream of the S-duct inlet plane) }\right. \\ S S M & =\text { Second Switching Mode } \\ S V M & =\text { Second Vertical Mode } \\ \text { std } & =\text { Standard deviation value of a temporal distribution }\end{array}$

\section{Superscripts}

$\begin{array}{ll}u & =\text { Lateral velocity field } \\ v & =\text { Vertical velocity field } \\ V_{i p} & =\text { In-plane velocity field } \\ w & =\text { Stream-wise velocity field }\end{array}$

\section{Operators}

$$
\begin{array}{ll}
\langle\cdot\rangle & =\text { Time-average } \\
\cdot & =\text { Area-average } \\
\sigma \cdot & =\text { Standard deviation }
\end{array}
$$

\section{Introduction}

CONVOLUTED aero-engine intakes are used in embedded engine systems which are expected to power the next generation of aircraft. S-shaped intakes are often employed to deliver the air flow to such integrated power plants. Integrated engine configurations allow for more compact and efficient aircraft designs, and are of interest to novel civil configurations $[1,2]$. However complex intake configurations promote high levels of dynamic total pressure and swirl distortion which can adversely affect the engine stability [3]. Unsteady total pressure and swirl distortion are generated as a result of flow separations and secondary flows within the intake. The detrimental effect of total pressure distortion on the engine operability has been widely investigated [4-8]. The effect of swirl distortion has received relatively less attention since historically swirl-related issues were implicitly mitigated with the utilisation of inlet guide vanes and relatively simple intake designs [3]. However the adverse effect of swirl distortion on the compression system stability margin was demonstrated during the development of several air vehicles [3]. Previous studies 
highlighted the importance of dynamic distortion and in particular the local peak values upon the onset of engine instabilities $[6,9,10]$.

Previous research has investigated the distorted flow field associated with S-shaped intakes. Wellborn et al. [11] studied the flow through a diffusing $\mathrm{S}$-duct $\left(\mathrm{AR}=1.52, \mathrm{~L} / \mathrm{D}_{\mathrm{in}}=5.0, \mathrm{H} / \mathrm{L}=0.27\right.$, Fig. 1) with low-bandwidth instrumentation. A total pressure deficit was identified in the lower sector of the Aerodynamic Interface Plane (AIP), where a symmetric pair of counter-rotating vortices was observed. Berens et al. [12] performed a Detached Eddy Simulation (DES) of the flow field in an S-duct with similar offset ratio $H / L=0.28\left(A R=1.4, L / D_{\text {in }}=3.76\right)$ and highlighted the limitations of time-averaged data for the intake/engine compatibility assessment. Garnier [13] investigated the performance of active flow control in an $\mathrm{S}$-duct $\left(\mathrm{AR}=1.52, \mathrm{~L} / \mathrm{D}_{\mathrm{in}}=4.95, \mathrm{H} / \mathrm{L}=0.50\right)$ using 40 highbandwidth transducers. Separated flow was detected at the inner bend of the S-duct for the uncontrolled flow case. For a flow condition of $\mathrm{M}_{\mathrm{AIP}}=0.20$, the separation point was identified at a centreline co-ordinate of $\mathrm{s} / \mathrm{D}_{\mathrm{in}}=2.17$, and the separation-bubble length of approximately $1.35 \mathrm{D}_{\text {in }}$ was estimated based on the static pressure distribution along the walls. The unsteady reattachment point was associated with frequencies between $\mathrm{St}=0.48$ and 1.20. Zachos et al. [14] first applied Stereo Particle Image Velocimetry (SPIV) to characterise the swirl distortion at the outlet of two Sshaped intakes with the same non-dimensional geometrical parameters $\left(H / L, A R, L / D_{\text {in }}\right)$ as the configurations investigated by Garnier [13] and Wellborn et al. [11], respectively. The main difference between these two configurations was the centreline offset which was $\mathrm{H} / \mathrm{L}=0.50$ and $\mathrm{H} / \mathrm{L}=0.27$, respectively. The inlet Mach number ranged from 0.27 to 0.60 , with a concomitant variation of $\operatorname{Re}_{\mathrm{D}}$ between $5.9 \times 10^{5}$ and $13.8 \times 10^{5}$. The maximum fluctuations of the stream-wise velocity were found in the upper bounds of the mean-flow main loss region, and were linked to the unsteadiness of the separated shear layer. The swirl angle maximum fluctuations were found in the lower sector of the AIP where the mean-flow was relatively swirl-free, and were linked to the unsteady secondary-flow vortices. The inlet Mach number showed a modest effect on the overall characteristics of both steady and fluctuating flow fields. The highly unsteady nature of the flow field resulted in significant levels of dynamic flow distortion. Peak values of Swirl Intensity (SI) as high as twice the mean value were reported. Notable excursions from the mean-flow twin swirl pattern were observed towards single swirling flow patterns rotating in either clockwise or anti-clockwise direction.

The importance of the dynamic flow distortion on the engine performance and operability [6] highlights the need for a better understanding of the flow features which promote deviations from the steady-state distortion levels. Proper 
Orthogonal Decomposition (POD) has been recently applied to identify the most energetic coherent structures in the flow within complex aero-engine intakes. MacManus et al. [15] performed a Delayed Detached Eddy Simulation (DDES) of the flow field in two S-ducts with different centreline offsets, which corresponded to the geometry investigated by Garnier [13] $(\mathrm{H} / \mathrm{L}=0.50)$ and a scaled version of the geometry investigated by Wellborn et al. [11] $(\mathrm{H} / \mathrm{L}=0.27)$. Two Mach numbers were simulated for each configuration, $\mathrm{M}_{\mathrm{AIP}}=0.18$ and $\mathrm{M}_{\mathrm{AIP}}=0.36$, which resulted in $\mathrm{Re}_{\mathrm{D}}$ of approximately $1.1 \times 10^{6}$ and $1.7 \times 10^{6}$ respectively. The Proper Orthogonal Decomposition (POD) was applied to the computed total-pressure field at the AIP. For both configurations, the dominant coherent structures consisted of a lateral and a vertical oscillation of the main loss region. It was proposed that the lateral oscillation was associated with the secondary flows, while the vertical perturbation was related to the unsteadiness of the centreline diffusiondriven separation. For the low offset duct $(\mathrm{H} / \mathrm{L}=0.27)$, the spectral analysis showed that the lateral and vertical perturbations were associated with frequencies of $\mathrm{St}=0.35$ and $\mathrm{St}=0.70$, respectively, for both $\mathrm{M}_{\mathrm{AIP}}=0.18$ and $\mathrm{M}_{\mathrm{AIP}}=0.36$. For the high offset duct $(\mathrm{H} / \mathrm{L}=0.50)$ at $\mathrm{M}_{\mathrm{AIP}}=0.18$, the frequencies associated with the lateral and vertical perturbations were $\mathrm{St}=0.55$ and $\mathrm{St}=0.85$, respectively. At high Mach number $\left(\mathrm{M}_{\mathrm{AIP}}=0.36\right)$ the spectrum associated with the vertical oscillation showed a more broadband spectral content.

Gil-Prieto et al. [16] used SPIV to characterise the distorted swirl pattern at the outlet of the same S-duct configurations investigated by Zachos et al. [14], with two different centreline offset values of $\mathrm{H} / \mathrm{L}=0.50$ and 0.27 . POD was applied on the three-component velocity vector at the AIP to identify the most-energetic coherent structures of the flow. The dominant coherent structures were the so-called switching and vertical perturbation modes. The switching mode promoted the swirl switching mechanism, by which one of the Dean vortices observed in the mean flow alternately dominates the flow field [17]. The swirl switching mode was previously observed by Kalpakli Vester et al. [18], who used Time-Resolved SPIV (TR-SPIV) for the measurement of the air-flow velocity field downstream a $90^{\circ}$ non-diffusing bend for two geometries with different curvature ratios of $\gamma=0.14$ and $\gamma=0.39$, at a $\operatorname{Re}_{\mathrm{D}}=2.3 \times 10^{4}$. The associated modal distribution for the stream-wise velocity resembled to the lateral perturbation reported by MacManus et al. [15] for the total pressure field, and represented a lateral modulation of the primary loss region, which followed the movement of the dominant vortex [16]. The vertical mode predominately represented a perturbation of the vertical velocity field. This mode also described a vertical modulation of the main loss region, which resembled to the vertical perturbation of the total pressure field reported by MacManus et al. [15]. Gil-Prieto et al. [16] also assessed the impact of the most-energetic coherent structures on the dynamic swirl distortion 
characteristics. The switching mode was found to be responsible for most of the bulk swirl events associated with the peak Swirl Intensity (SI) values, particularly in the high offset duct $(\mathrm{H} / \mathrm{L}=0.50)$. The vertical mode was associated with most of the twin swirl events for both configurations. This indicated the importance of these coherent structures in the swirl distortion pattern.

The aim of the present investigation is to use DDES simulations to expand the understanding of the most-energetic coherent structures in the S-duct flow field. The present study follows on from the experimental work by Gil-Prieto et al. [16] with a more detailed investigation of the flow field coherent structures. The DDES results are validated against SPIV measurements. The DDES simulations are time resolved and therefore permit the spectral analysis of the coherent structures observed with the temporally under-resolved SPIV results. The symmetry plane velocity field is investigated in order to identify the origin of the perturbations observed at the AIP which are responsible for the flow distortion characteristics. This is of prime importance for the design of flow control devices. A novel multi-plane POD based on both the AIP and symmetry plane velocity fields is conducted for this purpose.

\section{Methodology}

\section{A. Studied case}

The S-duct geometry is a scaled-down version of the geometry investigated by Garnier [13].The S-duct has a circular cross-section, and the main geometrical parameters are an area ratio of $A R=1.52$, a length of $L / D_{\text {in }}=4.95$ and a centreline offset of $\mathrm{H} / \mathrm{L}=0.50$ (Fig, 1). The $\mathrm{S}$-duct centreline is composed of two consecutive $52^{\circ}$ arcs with curvature ratios $\left(\mathrm{R}_{\mathrm{in}} / \mathrm{R}_{\mathrm{c}}\right)$ of $\gamma=0.16$. The flow condition is determined by the Mach number at the reference plane which is located $0.9 \mathrm{D}_{\text {in }}$ upstream of the S-duct inlet. The computational and experimental results presented in this work correspond to a reference Mach number of $\mathrm{M}_{\mathrm{ref}}=0.27$, which is associated with a $\mathrm{Re}_{\mathrm{D}}=7.1 \times 10^{5}$. The same S-duct geometry at $\mathrm{M}_{\mathrm{ref}}=0.27$ was tested by Zachos et al. [14], Gil-Prieto et al. [16] and Tanguy et al. [19].

6

American Institute of Aeronautics and Astronautics 


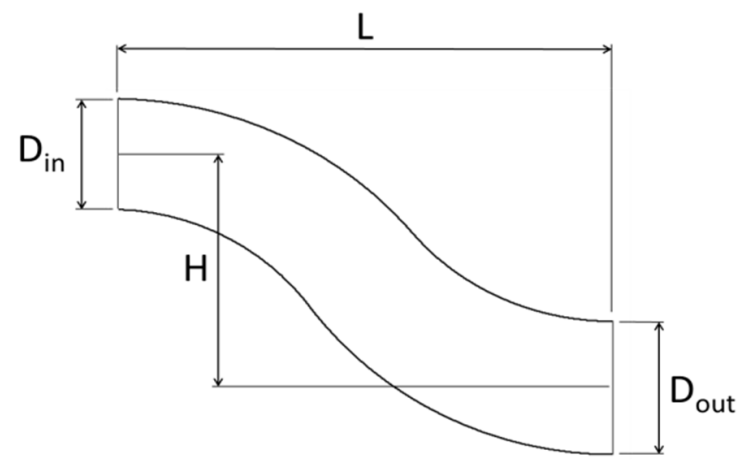

Fig. 1 Schematic definition of the S-duct geometrical parameters

\section{B. Stereo Particle Image Velocimetry experiment}

A detailed description of the experimental facility is reported by Zachos et al. [14], and only the key aspects of the experiment are reported here. A borosilicate glass, transparent section is placed downstream of the S-duct to permit optical access for both laser and cameras. The measurements are performed at the AIP which is located $0.24 \mathrm{D}_{\text {in }}$ downstream of the S-duct outlet. A single-stage centrifugal fan is used to control the mass-flow rate. The rig is calibrated to provide the required Mach number at the reference plane $\left(0.9 \mathrm{D}_{\text {in }}\right.$ upstream of the $\mathrm{S}$-duct inlet). The Mach number uncertainty at the flow condition considered in the present work is $0.27 \pm 0.01$. The boundary layer measured at the reference plane showed values of displacement thickness and shape factor of $8.2 \times 10^{-3} \mathrm{D}_{\text {in }}$ and 1.43 , respectively.

The SPIV system and methods used to obtain the three components of the velocity are the same as reported by Tanguy et al. [19]. The seeding particles were illuminated with a dual cavity, frequency-doubled Nd:YAG laser with a wave-length of $532 \mathrm{~nm}$ and a maximum power of $200 \mathrm{~mJ}$ per pulse. The laser light sheet was delivered by an articulated light arm which provides a light sheet with a thickness of approximately $2 \mathrm{~mm}$. The seeding particles were made of Di-Ethyl-Hexyl Sebacate (DEHS) and the estimated diameter of the particles was $1 \mu \mathrm{m}$. Two TSI PowerView Plus 8MP cameras were used in a stereoscopic configuration with an approximately $45^{\circ}$ off-axis arrangement. The acquisition rate was approximately $3.5 \mathrm{~Hz}$. TSI Insight $4 \mathrm{G}^{\mathrm{TM}}$ software was used for the calibration of the cameras, the acquisition and the processing of the images. About 14000 velocity vectors were obtained at the AIP, which resulted in a spatial resolution of $1.1 \mathrm{~mm}\left(0.007 \mathrm{D}_{\text {out }}\right)$. A disparity correction was applied to account for the potential misalignment between the laser light sheet and the calibration target. The SPIV measurements uncertainty was estimated with the procedure proposed by Raffel et al. [20], which takes into account the particle image displacement, particle image diameter, seeding density, quantization level and background noise. The overall uncertainty was 
approximately $6 \%$ and $8 \%$ for the in-plane and out-of-plane components of the velocity, respectively. A dataset of 1000 snapshots was considered to be sufficient to provide statistically converged results as reported by Zachos et al. [14].

\section{Delayed Detached Eddy Simulation}

The numerical computation is performed using a Detached Eddy Simulation (DES), for which the Unsteady Reynolds Averaged Navier-Stokes (URANS) equations are applied in the boundary layer, while the Large Eddy Simulation (LES) method is employed in the highly unsteady regions away from the wall [21]. The Delayed version of the DES (DDES) ensures the boundary layer is resolved with the URANS formulation, and is used to prevent gridinduced separation problems [22]. The k- $\omega$ SST model was chosen for the URANS turbulence modelling. A pressure based solver with a segregated PISO scheme was used. The momentum, density, energy and turbulence equations were spatially discretised with a third order MUSCL scheme, while the pressure equations were solved with a second order discretisation scheme. The temporal formulation was based on a second order implicit scheme. The ideal gas equation was used and the Sutherland law was chosen to model the air viscosity dependence upon the temperature.

The inlet total pressure profile was measured at the reference plane located $0.9 \mathrm{D}_{\text {in }}$ upstream of the S-duct inlet, using low bandwidth pressure probes. However this profile could not be applied directly as the inlet boundary condition for the computational domain, since this region was affected by the pressure gradient established in the first bend of the S-duct. A total pressure profile was applied $2 \mathrm{D}_{\text {in }}$ upstream of the S-duct inlet in order to match the experimental profile measured at the reference plane. The experimental boundary layer showed a displacement thickness of $8.2 \times 10^{-3} \mathrm{D}_{\text {in }}$ and a shape factor of 1.43 . The DDES solution predicted a boundary layer with similar values of displacement thickness and shape factor of $8.4 \times 10^{-3} \mathrm{D}_{\text {in }}$ and 1.42 , respectively. The experimental total temperature value of approximately $290 \mathrm{~K}$ was also imposed at the inlet of the computational domain. A uniform static pressure boundary condition was applied at the outlet of the domain in order to match the experimental mass flow rate. The outlet of the domain was extended $3 \mathrm{D}_{\text {in }}$ to remove any influence of the uniform boundary condition assumption on the solution.

A baseline structured mesh of 5 million nodes was generated with an H-grid structure in the centre of the S-duct section and an O-grid structure around the walls (Fig. 2). The mesh was refined near the walls in order to ensure the $\mathrm{y}+$ was smaller than 1 over the full domain, with an expansion ratio off the wall of 1.05 . The number of nodes in each cross section is approximately $11 \mathrm{k}$, and the number of cross sections along the domain is approximately 450 . The 
$2 \times 2 \times 2$ determinant is obtained at each mesh cell as the normalised determinant of the Jacobian matrix. A value of 1 represents a perfect cube while a value of 0 reflects a totally inverted cube with negative volume, and values above 0.3 are usually recommended [23]. For the mesh used in this investigation the determinant $2 \times 2 \times 2$ was greater than 0.83 over the domain which indicates a good quality mesh. The time step was set to $\Delta \mathrm{t}=1.2 \times 10^{-5} \mathrm{~s}$ for the $\mathrm{M}_{\mathrm{ref}}=0.27$ case considered in the present work, which corresponds to a non-dimensional time step of approximately $\Delta \mathrm{t} / \mathrm{t}_{\mathrm{c}}=1.53 \times 10^{-3}$. The convective time $t_{c}$ is based on the S-Duct centreline length and the inlet centreline stream-wise velocity. The DDES computation was initialised from a converged RANS simulation. Each time step was solved with 20 sub-iterations, which resulted in maximum residuals of the order of $10^{-6}$ for the continuity equation at the end of each time-step computation. The first $115 t_{c}$ of the unsteady DDES simulation are not considered for the analysis to remove any effect of the transition between the RANS and DDES solutions [15]. This is a conservative approach compared to the $10 \mathrm{t}_{\mathrm{c}}$ discarded by Berens et al. [12]. The statistical convergence of the flow field was assessed for different simulated times, which ranged from $20 \mathrm{t}_{\mathrm{c}}$ to $50 \mathrm{t}_{\mathrm{c}}$. The $\mathrm{SI}_{\text {mean }}, \mathrm{SI}_{\mathrm{std}}$ and $\mathrm{SI}_{\max }$ values were approximately the same for $20 \mathrm{t}_{\mathrm{c}}$ and $50 \mathrm{t}_{\mathrm{c}}$, with differences of the order of $0.01^{\circ}$. The results presented in this work are based on $50 \mathrm{t}_{\mathrm{c}}$.

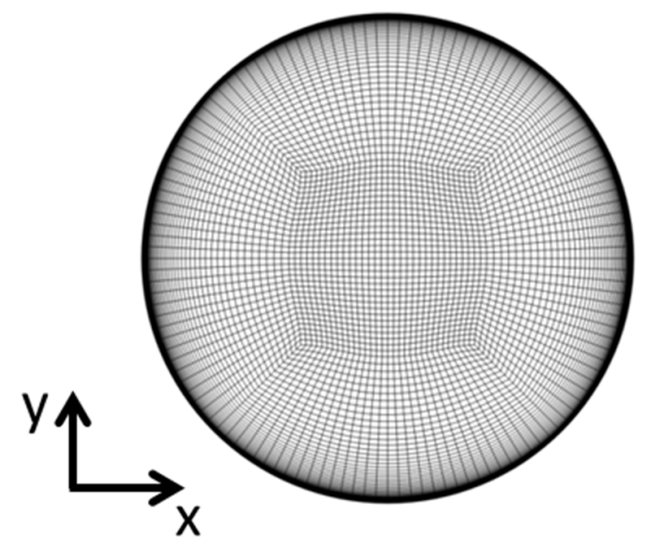

Fig. 2 Cross section mesh topology

Three meshes of 2.5, 5 and 10 million nodes respectively were considered to assess the sensitivity of the DDES unsteady solution to the mesh spatial resolution. The number of nodes in each direction was multiplied by a constant factor of 1.3 to generate the different meshes. The number of nodes in each cross section was about $7 \mathrm{k}, 11 \mathrm{k}$ and $18 \mathrm{k}$ for the coarse, medium and fine meshes, respectively. The number of cross sections along the domain was approximately 350,450 and 550, respectively. A mesh sensitivity study was available for the same geometry at a flow 
condition of $\mathrm{M}_{\mathrm{ref}}=0.60$. The conclusions from that study are presented here, and are expected to be also appropriate for the $\mathrm{M}_{\mathrm{ref}}=0.27$ simulation used in the present investigation, where the requirements are generally less stringent. Hence the mesh sensitivity was done with the results of the DDES simulation for these three meshes at $\mathrm{M}_{\mathrm{ref}}=0.60$, with a time step of $\Delta t=6 \times 10^{-6} \mathrm{~s}$, which equates to $\Delta t / t_{c}=1.86 \times 10^{-3}$. The same values of $P_{\text {mean }}$ and $P R_{\text {std }}$ were obtained for the three meshes considered. The $\mathrm{SI}_{\text {mean }}$ value was $10.2^{\circ}, 9.9^{\circ}$ and $9.3^{\circ}$ for the coarse, medium and fine meshes, respectively. The corresponding values of $\mathrm{SI}_{\text {std }}$ were $1.9^{\circ}, 1.8^{\circ}$ and $1.5^{\circ}$, while for $\mathrm{SI}_{\max }$ the values were $17.5^{\circ}, 16.8^{\circ}$ and $15.7^{\circ}$. This indicates a limited impact of the mesh spatial resolution on the swirl distortion characteristics. The impact of the time-step choice was also assessed at $\mathrm{M}_{\mathrm{ref}}=0.60$, and the DDES simulation with the medium mesh was computed at a doubled time-step of $\Delta \mathrm{t}=1.2 \times 10^{-5} \mathrm{~s}$, which equates to $\Delta \mathrm{t} / \mathrm{t}_{\mathrm{c}}=3.72 \times 10^{-3}$. For this coarser time step, the $\mathrm{SI}_{\text {mean }}, \mathrm{SI}_{\text {std }}$ and $\mathrm{SI}_{\max }$ values are approximately $9.8^{\circ}, 1.8^{\circ}$ and $16.5^{\circ}$, respectively. This indicates a relatively minor dependency of the swirl distortion characteristics upon the time-step choice. The results presented in this work are at $\mathrm{M}_{\mathrm{ref}}=0.27$, and are obtained using the 5 million nodes medium mesh with a $\Delta \mathrm{t}=1.2 \times 10^{-5} \mathrm{~s}\left(\Delta \mathrm{t} / \mathrm{t}_{\mathrm{c}}\right.$ of $\left.1.53 \times 10^{-3}\right)$.

The DDES approach switches between RANS and LES methods based on the value of a blending function, $\mathrm{f}_{d}$ [24], so that RANS is activated when $f_{d}=0$, while the LES formulation is applied when $f_{d}=1$. Ideally RANS should be applied within the boundary layers while LES should be activated in those regions away from the wall where large scale structures are expected to occur [22]. This has been checked for all the simulations performed in this investigation, including those required for the grid and time step sensitivity. An example of the blending function distribution at the AIP and symmetry plane is provided in Fig. 3, for the simulation at $\mathrm{M}_{\mathrm{ref}}=0.27$ with the medium mesh.

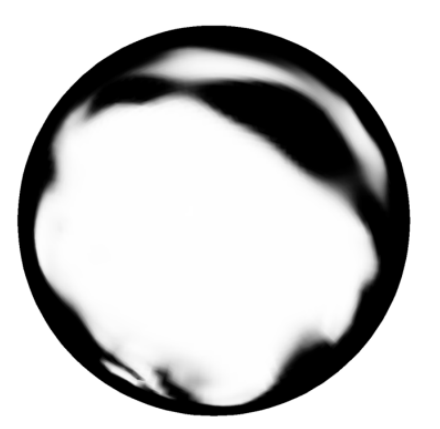

(a) AIP

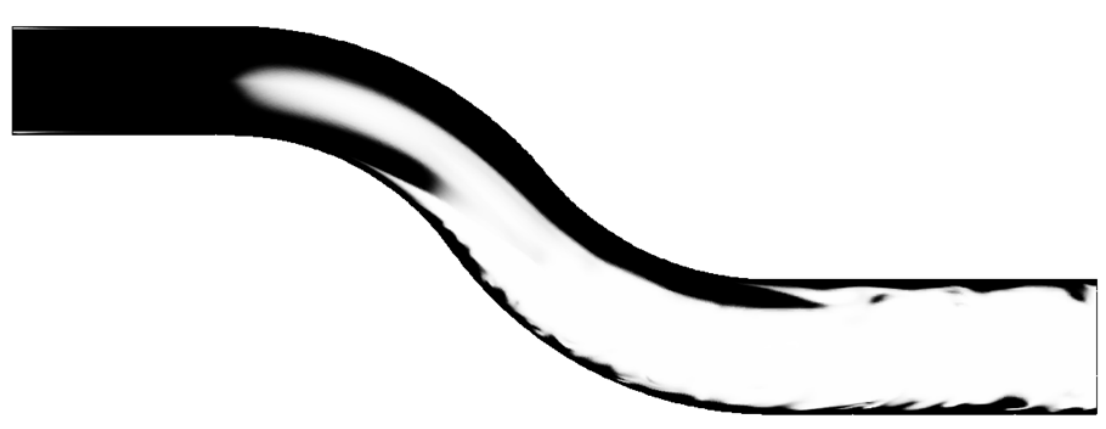

(b) Symmetry plane

Fig. 3 Sample time-step showing the DDES blending function distribution $\left(f_{d}=0\right.$ : black, $f_{d}=1$ : white) 


\section{Proper Orthogonal Decomposition}

Turbulent flows are characterised by the presence of coherent structures which are obscured by small-scale turbulent fluctuations. Coherent structures are large scale flow features which often account for most of the essential flow mechanisms [25]. The POD permits the identification of the most-energetic coherent structures of the flow field [26], and has been applied in a wide range of applications including the flow in curved pipes [18] and S-ducts [16,27]. The POD of the velocity vector field, $\vec{V}$, finds an orthonormal set of bases, $\vec{\Phi}_{\imath}=\left\{\Phi_{i}^{u}, \Phi_{i}^{v}, \Phi_{i}^{w}\right\}$, which are invariant with time. These functions are usually referred to as POD modes and represent flow features which are orthonormal to each other [26]. Each of the POD modes has an associated temporal coefficient, $a_{i}(t)$, so that the contribution from each flow feature to the original flow field is $a_{i}(t) \overrightarrow{\Phi_{l}}$. Therefore $a_{i}(t)$ represents the instantaneous weight of each flow feature at the different instants of time. The temporal coefficients are uncorrelated to each other, so that the different flow features described by the POD modes occur uncorrelated in time [26]. The POD representation of the original velocity field can be obtained as the linear sum of a finite number $k$ of modal contributions [28] (Eq. 1).

$$
\vec{V}_{k}=\sum_{j=1}^{k} a_{j}(t) \vec{\Phi}_{J}(x, y)
$$

The variance of the temporal coefficients, $\left\langle a_{j}{ }^{2}\right\rangle$, represents the contribution of each POD mode to the areaaveraged mean kinetic energy, $\langle\overline{K E}\rangle[28]$. The modes are then ordered by the associated $\langle\overline{K E}\rangle$ contribution. This permits an optimum representation in terms of kinetic energy in the sense that, for a given number of terms in the series, the POD maximises the kinetic energy content in the reconstruction (Eq. 2) [28]. The kinetic energy of the original flow field $\langle\overline{K E}\rangle$ can be obtained as the sum of the contribution from all the modes. In the present investigation the POD has been implemented with the method of snapshots, developed by Sirovich [29].

$$
\langle\overline{K E}\rangle_{k}=\sum_{j=1}^{k}\left\langle a_{j}^{2}\right\rangle
$$

\section{Results}

\section{A. Experimental validation}

The mean velocity field for the DDES solution is compared with the SPIV results at the same inlet Mach number of $\mathrm{M}_{\mathrm{ref}}=0.27$ (Fig. 4). The DDES solution has been linearly interpolated, using the Delaunay triangulation method, into the locii of the SPIV data points for a consistent comparison. The region of low stream-wise velocity is well captured in the DDES solution (Fig. 4a) compared with the SPIV data (Fig. 4e). The minimum values of stream-wise 
velocity in the loss region are $\langle w\rangle /\left\langle\bar{w}_{A I P}\right\rangle=0.73$ and 0.75 for DDES and SPIV, respectively. The DDES mean vertical velocity distribution (Fig. 4b) is also well predicted compared to the SPIV results (Fig. 4f). The pitch-down regions near the walls at both sides of the symmetry plane are slightly under-predicted by the DDES. The minimum values are $\langle v\rangle /\left\langle\bar{w}_{A I P}\right\rangle=-0.15$ and -0.19 for DDES and SPIV, respectively. The pitch-up central region is well matched, with a maximum value of $\langle v\rangle /\left\langle\bar{w}_{A I P}\right\rangle=0.13$ for both DDES and SPIV, respectively. The DDES mean lateral velocity field (Fig. 4c) agrees well with the SPIV measurements (Fig. 4g). The two opposite-sign regions of high lateral velocity at the bottom and top of the AIP are also well predicted by the DDES. The maximum absolute values of lateral velocity at the lower sector are $\langle u\rangle /\left\langle\bar{w}_{A I P}\right\rangle=0.13$ and 0.15 for DDES and SPIV, respectively. The corresponding values at the top regions are $\langle u\rangle /\left\langle\bar{w}_{A I P}\right\rangle=0.06$ and 0.07 . The lateral and vertical velocity fields result in the well-known symmetric pair of vortices, observed for both DDES (Fig. 4d) and SPIV (Fig. 4h) data. The DDES also predicts a pair of regions of high lateral velocity near the wall at the top of the AIP (Fig. 4c), which results in a secondary pair of vortices (Fig. 4d). These small vortical structures are however not revealed by the SPIV data (Fig. 4h). One of the main flow variables of interest in S-duct research is the swirl angle, due the destabilising effect the swirling flow can have on the downstream components of the engine [3]. The characteristic mean-flow pair of swirling regions at the AIP which are associated with the presence of the two counter-rotating vortices is observed for both DDES (Fig. 4d) and SPIV (Fig. 4h). In general, the measured data has very low noise levels. Measurement noise is only present for the lateral velocity measurements in regions very close to the wall (Fig. 4g) and are due to reflections of the laser light sheet. Overall, there is a good agreement between numerical and experimental time-averaged data. To further quantify the agreement between DDES and SPIV data, the mean flow profile of the three components of the velocity along the symmetry axis of the AIP is assessed (Fig. 5). Both DDES and SPIV results show similar mean flow profiles for the three components of the velocity (Fig. 5).

The highly unsteady nature of the S-duct AIP flow field is well recognised [12,14-16]. Therefore it is also of interest to compare the calculated DDES fluctuating velocity field with SPIV data. The standard-deviation of the three components of the velocity and the swirl angle at the AIP for DDES and SPIV are compared (Fig. 6). It is important to highlight that the DDES flow field was acquired at a frequency of $27.8 \mathrm{kHz}$ for approximately $50 \mathrm{t}_{\mathrm{c}}(0.40 \mathrm{~s})$, which are sufficient to provide statistically converged results (see Section II. C). In contrast, the SPIV measurements are temporally under-resolved with an acquisition frequency of $3.5 \mathrm{~Hz}$ for $286 \mathrm{~s}$ to provide 1000 snapshots for the statistical analysis, which ensures statistically converged results [14]. For statistically converged data sets, the flow 
field statistics should be independent of the acquisition frequency, and therefore the DDES and SPIV statistics are comparable. The extent of the region of greatest stream-wise velocity fluctuations is in good agreement between DDES (Fig. 6a) and SPIV data (Fig. 6e). This region corresponds to the upper boundary of the mean-flow shear layer. The maximum value is approximately $\sigma_{w} /\left\langle\bar{w}_{A I P}\right\rangle=0.22$ for both DDES and SPIV. However, DDES data do not show the small region of high stream-wise velocity fluctuations observed in the SPIV measurements at the top of the AIP (Fig. 6e). The greatest vertical velocity fluctuations occur at the centre of the section for both DDES (Fig. 6b) and SPIV data (Fig. 6f). However the fluctuation levels are slightly over-predicted in the DDES solution, for which the maximum values are $\sigma_{v} /\left\langle\bar{w}_{A I P}\right\rangle=0.26$ compared to the SPIV value of 0.23 . The maximum lateral velocity fluctuations occur at the lower sector of the AIP for both DDES (Fig. 6c) and SPIV data (Fig. 6g). As for the vertical component of the velocity, the lateral velocity fluctuations are over-predicted by the DDES. The maximum values are $\sigma_{u} /\left\langle\bar{w}_{A I P}\right\rangle=0.28$ and 0.22 for DDES and SPIV, respectively. The regions of maximum lateral and vertical fluctuations occur as a result of the highly unsteady oscillation of the two vortices observed in the mean-flow field (Fig. 4h). The region of maximum swirl angle fluctuations is located at the lower sector of the AIP, both for DDES (Fig. 6d) and SPIV data (Fig. 6h). The fluctuations in swirl angle are also over-predicted by the DDES solution, with maximum levels of approximately $\sigma_{\alpha}=19^{\circ}$ which are greater than the SPIV peak values of about $16^{\circ}$. Nevertheless, there is generally good agreement in the overall distributions of the unsteady flow characteristics. To quantify the discrepancies between the DDES and SPIV results in terms of fluctuating flow field, the profile of the standard deviation of the three components of the velocity is assessed at the symmetry axis of the AIP (Fig. 5). The qualitative trend of these profiles is well captured by the DDES, even though some quantitative discrepancies can be identified. The maximum discrepancies for the stream-wise, vertical and lateral velocities are approximately $0.06\left\langle\bar{w}_{A I P}\right\rangle$ (Fig. 5a), 0.05 $\left\langle\bar{w}_{A I P}\right\rangle$ (Fig. 5b) and $0.06\left\langle\bar{w}_{A I P}\right\rangle$ (Fig. 5c), respectively.

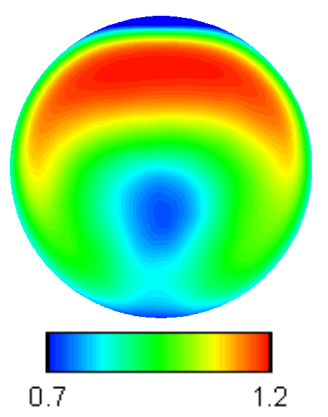

(a) $\langle w\rangle /\left\langle\bar{w}_{A I P}\right\rangle$,DDES

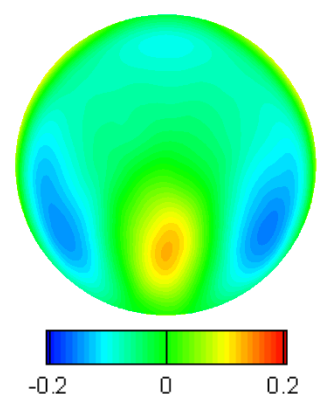

(b) $\langle v\rangle /\left\langle\bar{w}_{A I P}\right\rangle$, DDES

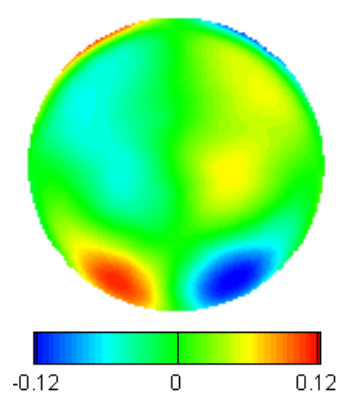

(c) $\langle u\rangle /\left\langle\bar{w}_{A I P}\right\rangle$, DDES

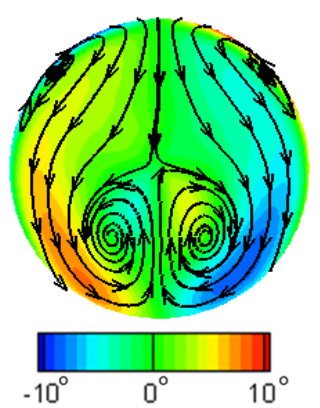

(d) $\langle\alpha\rangle$, DDES 


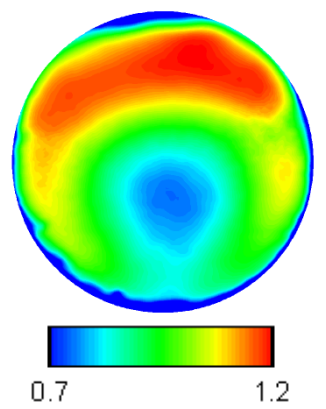

(e) $\langle w\rangle /\left\langle\bar{w}_{A I P}\right\rangle$, SPIV

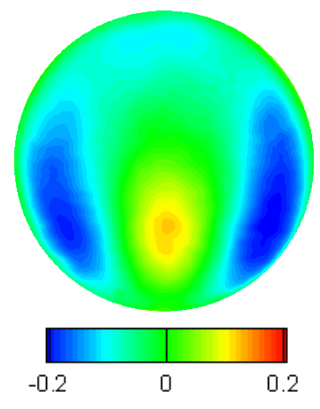

(f) $\langle v\rangle /\left\langle\bar{w}_{A I P}\right\rangle$, SPIV

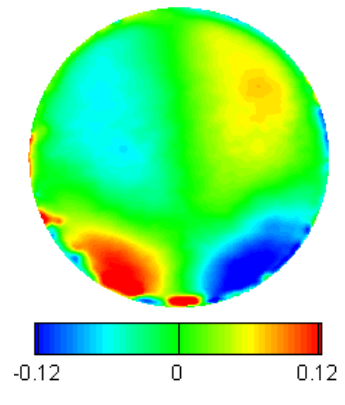

(g) $\langle v\rangle /\left\langle\bar{w}_{A I P}\right\rangle$, SPIV

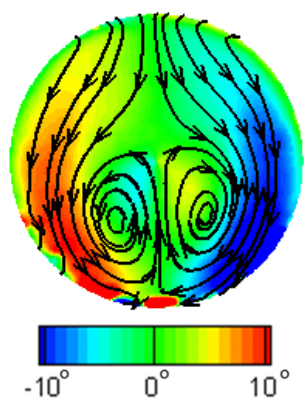

(h) $\langle\alpha\rangle$, SPIV

Fig. 4 Time-averaged flow field at the AIP at $M_{\text {ref }}=0.27$

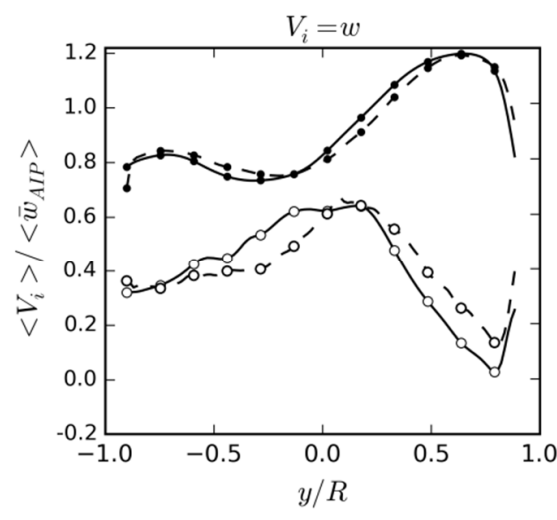

(a) Stream-wise velocity

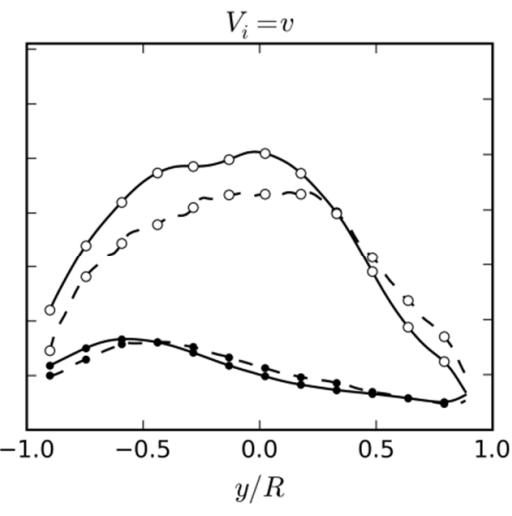

(b) Vertical velocity

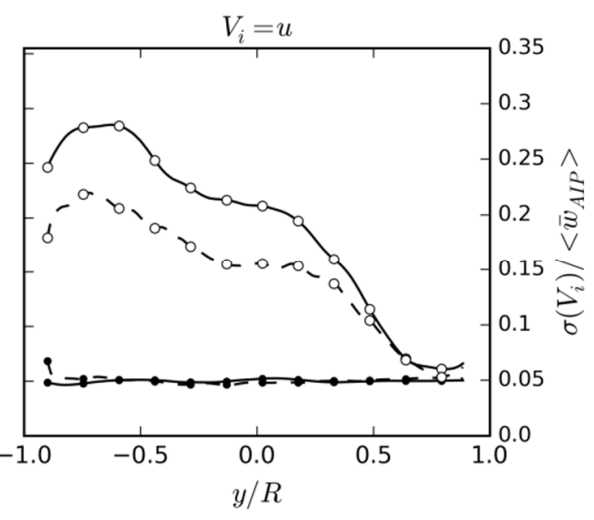

(c) Lateral velocity

Fig. 5 Velocity profiles along the symmetry axis of the AIP (DDES- solid line, SPIV- dashed line, unsteady

$$
\sigma\left(V_{i}\right) \text { - white circles, time-averaged }\left\langle V_{i}\right\rangle \text { - black circles) }
$$

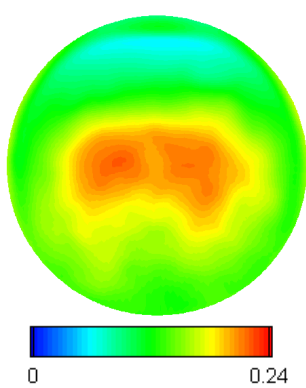

(a) $\sigma_{w} /\left\langle\bar{w}_{A I P}\right\rangle$, DDES

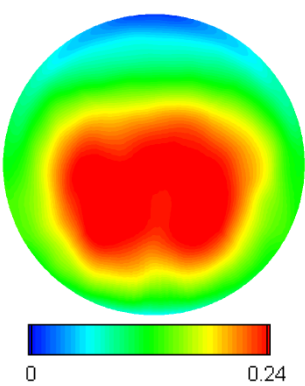

(b) $\sigma_{v} /\left\langle\bar{w}_{A I P}\right\rangle$, DDES

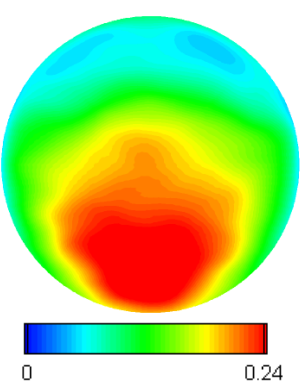

(c) $\sigma_{u} /\left\langle\bar{w}_{A I P}\right\rangle$, DDES

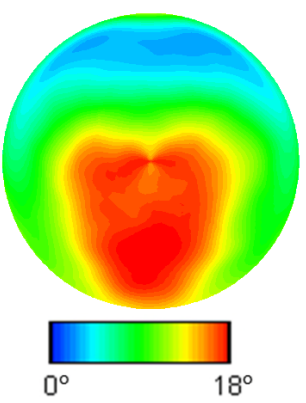

(d) $\sigma_{\alpha}$, DDES 


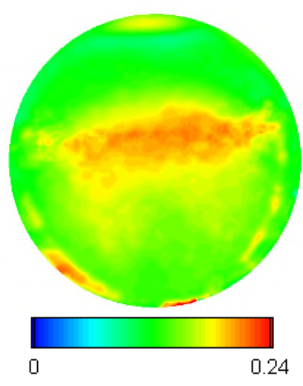

(e) $\sigma_{w} /\left\langle\bar{w}_{A I P}\right\rangle$, SPIV

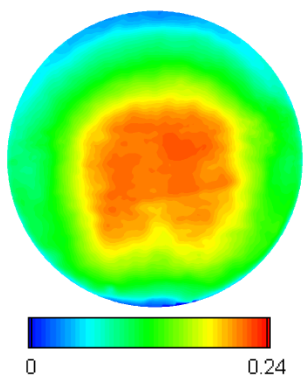

(f) $\sigma_{v} /\left\langle\bar{w}_{A I P}\right\rangle$, SPIV

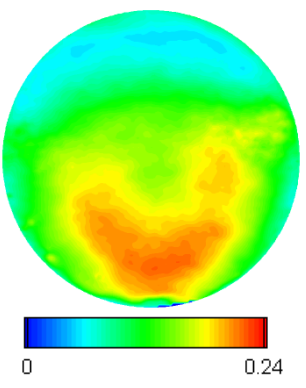

(g) $\sigma_{u} /\left\langle\bar{w}_{A I P}\right\rangle$, SPIV

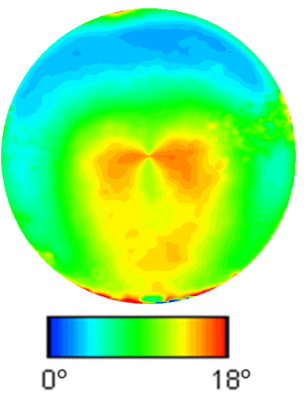

(h) $\sigma_{\alpha}$, SPIV

Fig. 6 Standard-deviation flow field at the AIP at $M_{\text {ref }}=0.27$

The Swirl Intensity (SI) distortion descriptor [3] is calculated for both DDES and SPIV data in order to quantify the differences between computational and experimental results. The AIP is discretised in 5 equal-area rings and 72 equi-spaced circumferential locations. The outer-most ring at $r / R=0.95$ is not considered in order to remove the potential effect of some spurious SPIV data near the wall as a result of the laser reflections. A single value of SI is then computed at each snapshot as the area-weighted average from the 4 considered rings. The SI statistics are in good agreement between DDES and SPIV data (Table 1). The mean and standard-deviation are matched within $1^{\circ}$ and $0.1^{\circ}$, respectively. The higher-order statistics such as the skewness [30] and kurtosis [31] are also in good agreement. Even the maximum and minimum SI values are very similar for both DDES and SPIV. Therefore the instantaneous SI predicted in the DDES solution shows similar mean and peak values, as well as similar probability distributions as indicated by the standard-deviation, skewness and kurtosis, compared to the SPIV measurements.

Table 1 Area-weighted Swirl Intensity statistics at $\mathbf{M}_{\text {ref }}=0.27$

\begin{tabular}{rcc} 
& DDES & SPIV \\
\cline { 2 - 3 } mean & $9.7^{\circ}$ & $8.7^{\circ}$ \\
std & $1.8^{\circ}$ & $1.7^{\circ}$ \\
skewness & 0.52 & 0.45 \\
kurtosis & 2.94 & 3.01 \\
$\max$ & $16.1^{\circ}$ & $14.9^{\circ}$
\end{tabular}


$\min$ $5.6^{\circ}$ $5.1^{\circ}$

\section{B. Coherent structures at the AIP}

POD [26] is applied to the three-component velocity vector at the AIP for both the CFD and SPIV data. For a consistent comparison the DDES solution is linearly interpolated using the Delaunay triangulation method into the loci of the SPIV data points prior to the POD computation. The POD permits the identification of the flow coherent structures. Mode 0 represents the mean flow and is referred to as Mean-Flow Mode (MFM), while the higher modes can be interpreted as perturbations to the mean [32]. The temporal coefficient associated with the MFM shows a high mean value with negligible oscillations. The temporal coefficients associated with the higher modes oscillate around the null mean-value. The modes are ordered in decreasing order of variance of the associated temporal coefficient. Therefore the higher the numbering of the mode the smaller is the perturbation impact upon the mean-flow. When POD is applied to the velocity vector the variance of the temporal coefficient coincides with the $\langle\overline{T K E}\rangle$ contribution of each mode to the overall flow field. Therefore the POD modes are ordered by $\langle\overline{T K E}\rangle$ content.

\section{DDES and SPIV coherent structures}

The four most-energetic coherent structures for the stream-wise and in-plane velocity fields as predicted by DDES are similar compared to SPIV data (Fig. 7). The so-called First Switching Mode (FSM) shows an antisymmetric distribution of the stream-wise velocity component with respect to the vertical symmetry plane both for DDES (Fig. 7a) and SPIV data (Fig. 7c). This mode shows a dominant swirling cell as revealed by the in-plane velocity pattern (Fig. 7b,d). Depending on the sign of the associated POD temporal coefficient the swirling cell perturbation rotates in either clock or anti-clock wise direction. This perturbation was previously reported by Gil-Prieto et al. [16], and shows the same in-plane velocity characteristics observed by Kalpakli Vester et al. [18] downstream of simpler, non-diffusing pipes with a single $90^{\circ}$ bend. This perturbation is responsible for the swirl switching mechanism by which alternatively one of the vortices observed in the mean-flow becomes dominant. Snapshots of the DDES in-plane velocity field reconstructed using only the FSM super-imposed on the time-averaged flow demonstrate the swirl switching mechanism (Fig. 8c,d). The extreme values of the associated POD temporal coefficient are considered to show the maximum deviation from the symmetric pair of Dean vortices observed in the mean flow. This in-plane velocity perturbation is associated with a circumferential oscillation of the low stream-wise velocity region which follows the swirl switching (Fig. 8a,b). For example, when the left vortex dominates the AIP flow field (Fig. 8c), it migrates 
towards a more central position while confining the other vortex towards the wall. The primary loss region then follows the movement of the dominant vortex (Fig. 8a).

The so-called First Vertical Mode (FVM) shows a symmetric distribution for the stream-wise velocity component with respect to the vertical symmetry plane both for DDES (Fig. 7e) and SPIV data (Fig. 7g). This mode predominately represents a perturbation of the vertical velocity field in the lower sector of the AIP (Fig. 7f,h), and is associated with a modulation of the vertical extent of the low stream-wise velocity region around its mean position. This perturbation was previously observed by Gil-Prieto et al. [16]. The effect of the FVM perturbation on the mean stream-wise velocity field is illustrated considering the reconstructed flow field with only the FVM and mean flow contributions (Fig. 8e,f). When the associated temporal coefficient is positive the main loss region extends upwards (Fig. 8e), while the opposite occurs for negative values for which the spoiled region becomes more confined to the lower wall (Fig. 8f). The effect on the in-plane flow is to modify the strength, position and area covered by the vortex pair (Fig. $8 \mathrm{~g}, \mathrm{~h})$. When $a_{F V M}(\mathrm{t})$ is positive the pair of vortices becomes stronger and move into a more centred position in the AIP (Fig. 8g). For a sufficiently low value of $a_{F V M}(\mathrm{t})$ the secondary kinetic energy associated with the vortices eventually vanishes and the in-plane topology is simply dominated by the general downward pitching flow expected at the exit of the duct (Fig. 8h).

First Switching Mode (FSM), DDES

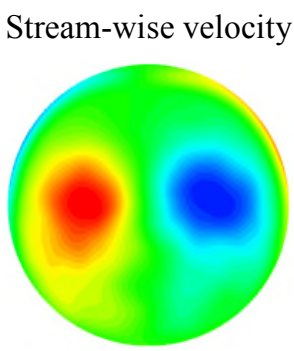

In-plane velocity

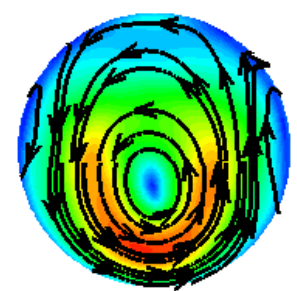

First Switching Mode (FSM), SPIV

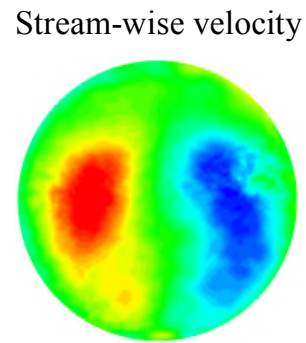

In-plane velocity

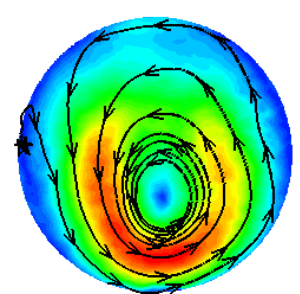

17

American Institute of Aeronautics and Astronautics 


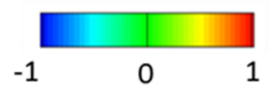

(a) $\Phi_{F S M}^{w}$

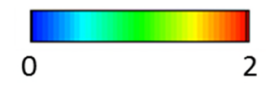

(b) $\Phi_{F S M}^{V_{i p}}$

First Vertical Mode (FVM), DDES

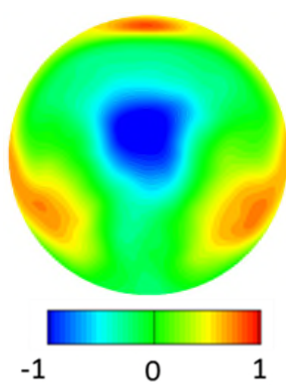

(e) $\Phi_{V M}^{W}$

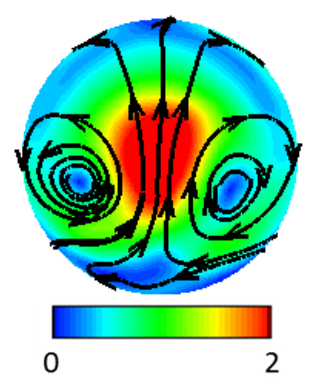

(f) $\Phi_{V M}^{V_{i p}}$

Second Switching Mode (SSM), DDES

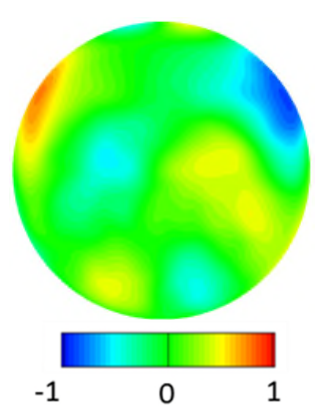

(i) $\Phi_{S S M}^{W}$

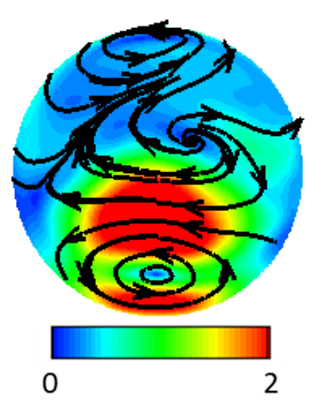

(j) $\Phi_{S S M}^{V_{i p}}$

Second Vertical Mode (SVM), DDES

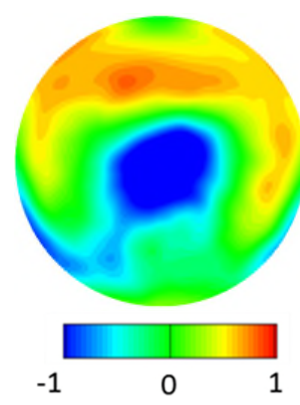

(m) $\Phi_{M F S M}^{W}$

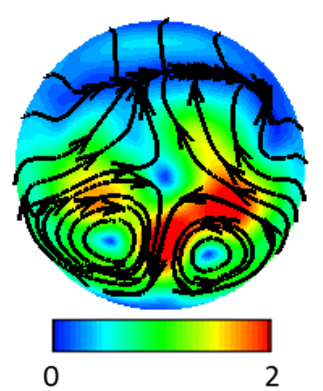

(n) $\Phi_{M F S M}^{V_{i p}}$

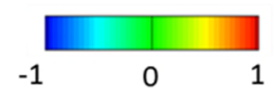

(c) $\Phi_{F S M}^{w}$

(d) $\Phi_{F S M}$

First Vertical Mode (FVM), SPIV

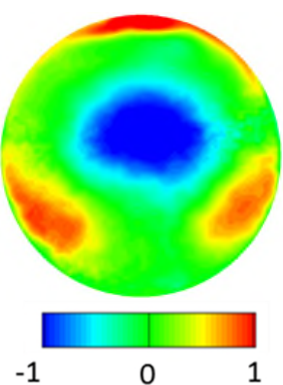

(g) $\Phi_{V M}^{W}$

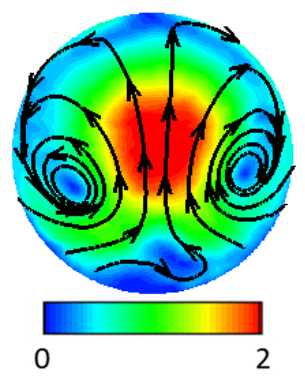

(h) $\Phi_{V M}^{V_{i p}}$

Second Switching Mode (SSM), SPIV

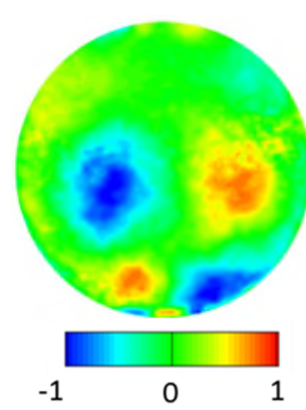

(k) $\Phi_{S S M}^{w}$

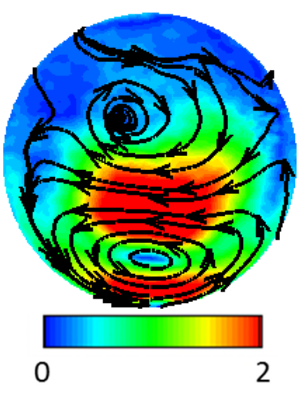

(1) $\Phi_{S S M}^{V_{i p}}$

Second Vertical Mode (SVM), SPIV

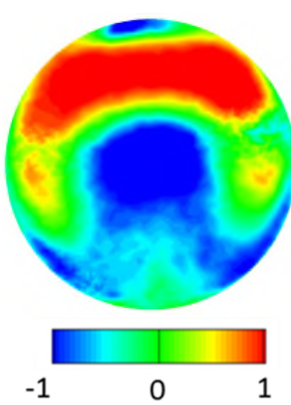

(o) $\Phi_{M F S M}^{W}$

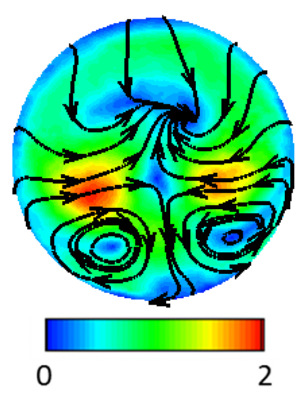

(p) $\Phi_{M F S M}^{V_{i p}}$

Fig. 7 Most-energetic POD modes for DDES (left) and SPIV (right) at $M_{\text {ref }}=0.27$

First Switching Mode (FSM)

Stream-wise velocity

$a_{F S M_{\text {max }}} \quad a_{F S M_{\text {min }}}$
In-plane velocity

$$
a_{F S M_{\max }} a_{F S M_{\text {min }}}
$$




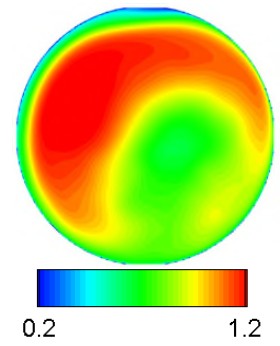

(a) $w_{M F M+F S M} /\left\langle\bar{w}_{A I P}\right\rangle$

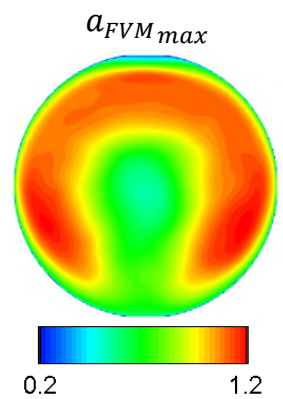

(e) $w_{M F M+F V M} /\left\langle\bar{w}_{A I P}\right\rangle$

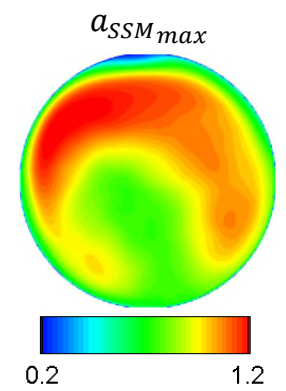

(i) $w_{M F M+S S M} /\left\langle\bar{w}_{A I P}\right\rangle$

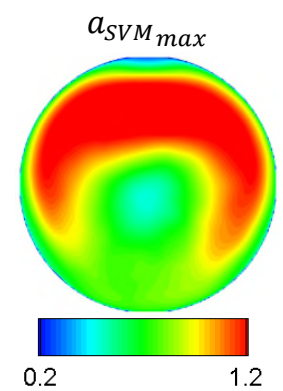

(m) $w_{M F M+S V M} /\left\langle\bar{w}_{A I P}\right\rangle$

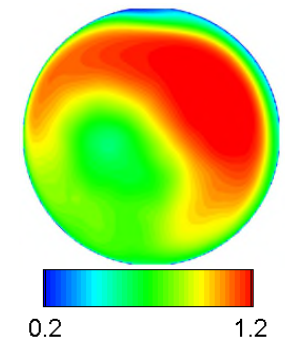

(b) $w_{M F M+F S M} /\left\langle\bar{w}_{A I P}\right\rangle$

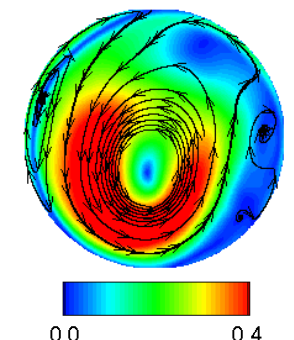

(c) $V_{i p_{M F M+F S M}} /\left\langle\bar{w}_{A I P}\right\rangle$

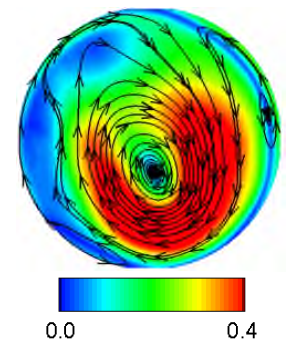

(d) $V_{i p_{M F M+F S M}} /\left\langle\bar{w}_{A I P}\right\rangle$

First Vertical Mode (FVM)

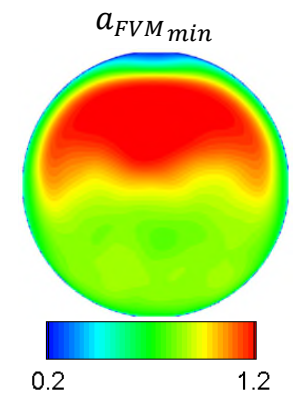

(f) $w_{M F M+F V M} /\left\langle\bar{w}_{A I P}\right\rangle$

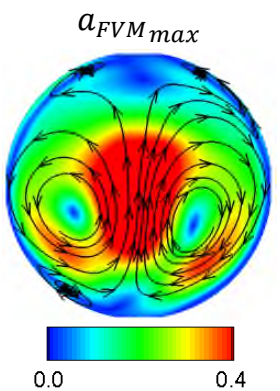

(g) $V_{i p_{M F M+F V M}} /\left\langle\bar{w}_{A I P}\right\rangle$

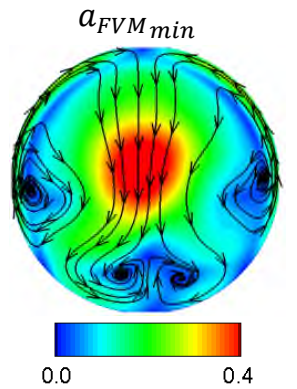

(h) $V_{i p_{M F M+F V M}} /\left\langle\bar{w}_{A I P}\right\rangle$

Second Switching Mode (FSM)

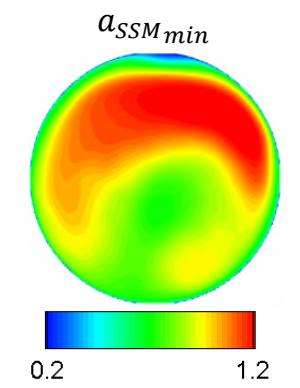

(j) $w_{M F M+S S M} /\left\langle\bar{w}_{A I P}\right\rangle$

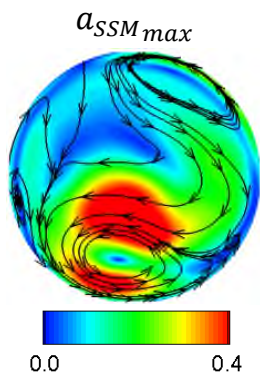

(k) $V_{i p_{M F M+S S M}} /\left\langle\bar{w}_{A I P}\right\rangle$

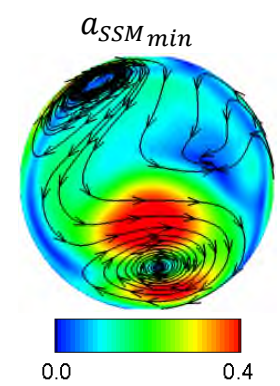

(1) $V_{i p_{M F M+S S M}} /\left\langle\bar{w}_{A I P}\right\rangle$

Second Vertical Mode (SVM)

Fig. 8 Snapshots of the isolated effect of the most-energetic modes on the mean flow (DDES, $\left.M_{\text {ref }}=0.27\right)$ 
The so-called Second Switching Mode (SSM) shows a dominant swirling cell at the lower sector of the AIP (Fig. $7 \mathrm{j}, 1)$. This perturbation promotes a swirl switching similar to that observed for the FSM. However, in this case the swirl switching occurs closer to the lower wall (Fig. 8k,1). The stream-wise component shows an antisymmetric distribution with respect to the vertical symmetry plane (Fig. 7i,k). A pair of opposite-sign regions is located at the centre of the AIP (Fig. 7i,k). An extra pair of opposite-sign regions is also present near the bottom wall, but in this case the sign is switched with respect to the centred pair of structures (Fig. 7i,k). This perturbation is associated with a lateral displacement of the main loss region position at the AIP (Fig. 8i,j). In contrast to the FSM, in this case the main loss region displacement is out-of-phase with the swirl switching. For example, when the left clockwise vortex dominates the lower sector of the AIP, it migrates to a more centred position (Fig. 8i). In this situation the main loss region appears tilted towards the left side of the AIP (Fig. 8c).

The so-called Second Vertical Mode (SVM) shows a very similar stream-wise velocity pattern compared to the mean flow distribution, which represents an out-of-phase oscillation between the mean high- and low- stream-wise velocity regions (Fig. 7m,o). The effect of this perturbation is to modulate the stream-wise velocity distortion between the high and low stream-wise velocity regions. When $a_{S V M}(\mathrm{t})$ is positive the gradient between the high and low velocity regions increases (Fig. 8m) compared to the mean flow, while the opposite occurs for negative $a_{S V M}(\mathrm{t})$ values (Fig. 8n). The associated in-plane streamlines pattern show a pair of counter-rotating vortical structures at the lower sector of the AIP (Fig. 7n,p). The effect upon the mean in-plane velocity field is a modulation of the magnitude of the secondary flows. When $a_{S V M}(\mathrm{t})$ is positive the secondary flows are reduced (Fig. 8o), while the mean-flow vortices are strengthened when $a_{S V M}(\mathrm{t})$ is negative (Fig. 8p). Therefore when this perturbation increases the gradient in the stream-wise velocity field at the AIP, the secondary flows are reduced, and vice versa.

Overall the most-energetic flow coherent structures are very similar for both DDES and SPIV data. However, there are some differences in the energetic content of the modal perturbations between the computational and experimental results (Table 2). The main difference between DDES and SPIV in terms of modal energetic content is found in the First Switching Mode (FSM). While the SPIV experimental data indicates a value of $0.78 \%$ compared to the areaaveraged kinetic energy accounted for by the mean flow $\langle\overline{K E}\rangle_{M F M}$, the value for the DDES solution is about $\langle\overline{T K E}\rangle_{F S M} /\langle\overline{K E}\rangle_{M F M}=1.69 \%$. The energy content of the Second Switching Mode (SSM) is also over-predicted by the DDES solution, with a value of $0.57 \%$ compared to the SPIV value of $0.27 \%$. In contrast, the DDES under-predicts the energetic content of the First Vertical Mode (FVM) and Second Vertical Mode (SVM) which show values of 
$0.80 \%$ and $0.34 \%$, respectively, compared to the $0.93 \%$ and $0.46 \%$ obtained for the SPIV data. Therefore even though the same fundamental structures are found for both numerical and experimental data, the actual energetic content of these flow structures is different. This is consistent with the discrepancies in the velocity field fluctuation levels previously observed between DDES and SPIV data (Fig. 6).

Table 2 Modal energy of the AIP velocity field POD at $M_{\text {ref }}=0.27$

\begin{tabular}{ccc}
\hline \hline \multicolumn{3}{c}{$\langle\overline{T K E}\rangle_{i} /\langle\overline{K E}\rangle_{M F M}(\%)$} \\
\hline \hline Mode $\boldsymbol{i}$ & DDES & SPIV \\
\hline FSM & 1.69 & 0.78 \\
FVM & 0.80 & 0.93 \\
SSM & 0.57 & 0.27 \\
SVM & 0.34 & 0.46 \\
\hline
\end{tabular}

\section{Spectral analysis}

The DDES simulations are time resolved and therefore can be used to further develop the understanding of the temporally under-resolved SPIV results through a spectral analysis of the POD temporal coefficients (Fig. 9). The Power Spectrum Density (PSD) of the POD temporal coefficients is computed using a Hanning window. The temporal coefficients associated with the computed First and Second Switching Modes (FSM and SSM) show a single spectral peak at about $\mathrm{St}=0.53($ Fig. $9 \mathrm{a}, \mathrm{c})$. The phase between $a_{F S M}(\mathrm{t})$ and $a_{S S M}(\mathrm{t})$ at this frequency $(\mathrm{St}=0.53)$ is computed and is approximately $90^{\circ}$. This suggests that the perturbations associated with the FSM and SSM are promoted by the same flow feature, which has a different impact on the AIP flow field depending on the particular instant of time. This is further developed in Section III D below, where the POD is applied simultaneously at the AIP and symmetry plane velocity fields. The FVM and SVM show a more broadband spectrum, even though a distinct peak can be identified around 1.06 (Fig. 9b,d). 


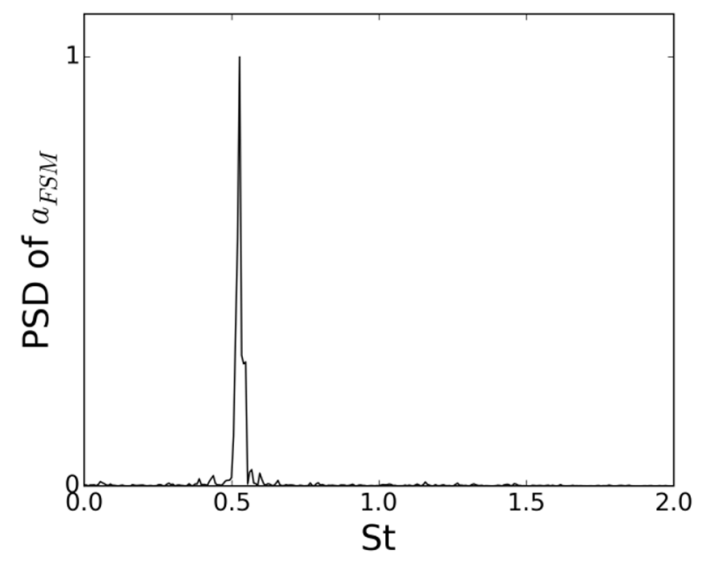

(a) First Switching Mode (FSM)

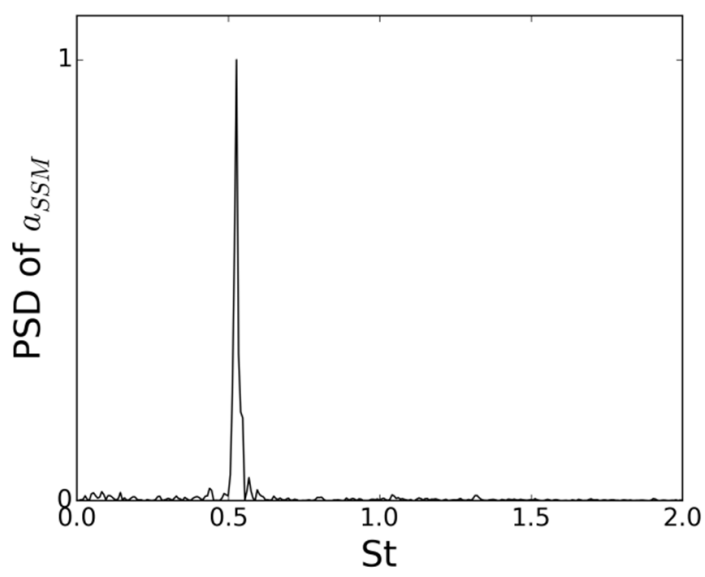

(c) Second Switching Mode (SSM)

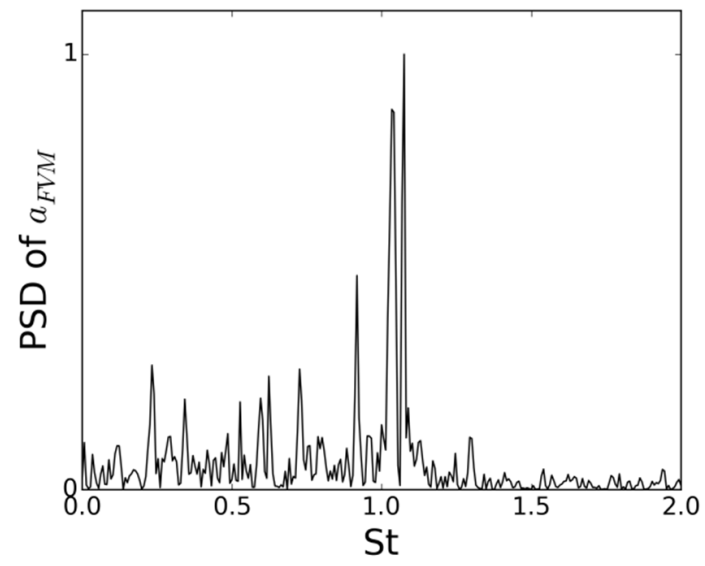

(b) First Vertical Mode (FVM)

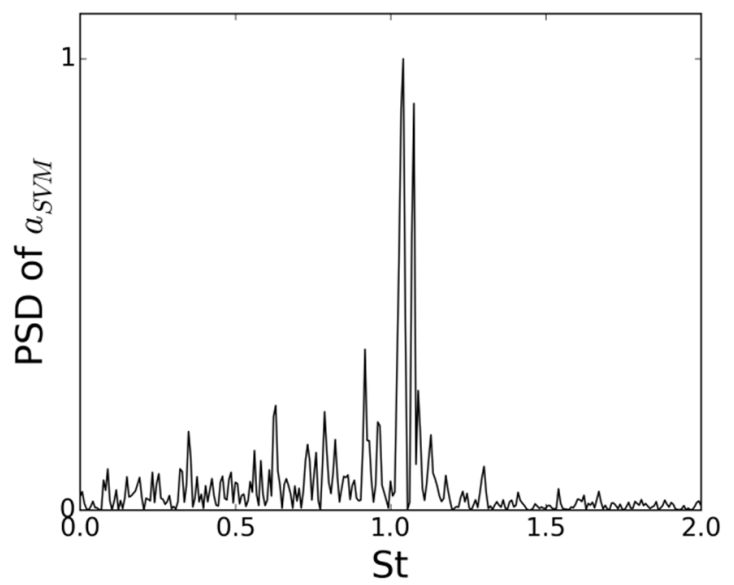

(d) Second Vertical Mode (SVM)

Fig. 9 Normalized PSD of the AIP velocity field POD temporal coefficients $\left(D D E S, M_{r e f}=0.27\right)$

The POD analysis of the velocity field at the AIP has identified the main coherent structures which are responsible for most of the flow field unsteadiness. These flow field fluctuations promote unsteady swirl distortion at the AIP which can result in maximum Swirl Intensity (SI) values of approximately twice $\mathrm{SI}_{\text {mean }}$ (Table 1). In addition, the unsteady swirl distortion pattern deviates from the well-known symmetric vortex pair, and multi-swirl structures as well as single-rotating cells are promoted [16]. This type of dynamic flow distortion is able to promote the onset of engine instabilities $[6,9,10]$. Previous studies have shown that both passive and active flow control devices can be used in S-duct intakes to stabilise the flow and to reduce the unsteadiness and dynamic distortion levels [13,19]. The impact of the flow control device strongly depends on the position within the S-duct. For example, passive flow control based on vortex generators is very sensitive to the location relative to the separation point at the first bend [19]. The design of an efficient flow control system depends on a good understanding of the origin of the AIP perturbations 
upon which the flow control device has to act. Within that context, it is useful to extend the analysis of the flow field and the POD modes at the AIP to also include the effect of the upstream flow. This is considered in the following sections through a combined assessment of the computed flow field at the AIP and symmetry plane.

\section{Symmetry plane flow field}

The mean flow at the symmetry plane is characterised by the presence of a separated flow region at the inner bend as indicated by the reversed flow (Fig. 10a). The mean position of the separation and reattachment points corresponds to saddle points, where the wall shear-stress is null [33]. The separation point at the wall corresponds to a centreline co-ordinate of $\mathrm{s} / \mathrm{D}_{\mathrm{in}}=2.0$, and the separation bubble length is approximately $1.7 \mathrm{D}_{\mathrm{in}}$. The average separation and reattachment points were also estimated experimentally with oil flow visualisations at the same flow conditions. The separation point in the experiment was observed at approximately the same position as predicted by the DDES solution. The separation bubble length is slightly under predicted by the DDES solution compared to the experimental value of $1.95 \mathrm{D}_{\text {in. }}$. The computed mean vertical velocity field shows positive values near the lower wall which is associated with the presence of the symmetric vortices (Fig. 10c). The mean lateral flow at the symmetry plane is effectively zero (Fig. 10e), which indicates the symmetry of the mean flow with respect to the symmetry plane. However lateral velocity fluctuations as high as $\sigma_{u} /\left\langle\bar{w}_{A I P}\right\rangle=0.36$ occur near the lower wall downstream the separation bubble (Fig. 10f). Fluctuations as high as $\sigma_{v} /\left\langle\bar{w}_{A I P}\right\rangle=0.36$ are also observed for the vertical velocity field downstream of the separation bubble (Fig. 10d), at the region where the mean-flow shear layer is located (Fig. 10a). The maximum fluctuations for the stream-wise velocity are approximately $\sigma_{w} /\left\langle\bar{w}_{A I P}\right\rangle=0.24$, and they occur along the mean-flow shear layer as well as near the lower wall just downstream the separation bubble (Fig. 10b). These results indicate that the maximum fluctuations of the velocity field originate just downstream of the separation bubble.
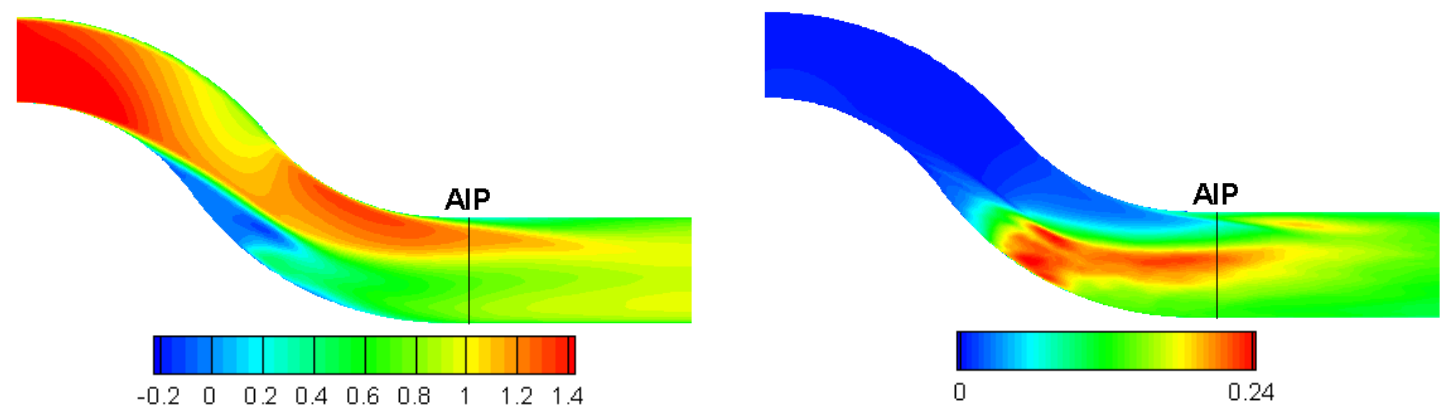

23

American Institute of Aeronautics and Astronautics 
(a) $\langle w\rangle /\left\langle\bar{w}_{A I P}\right\rangle$

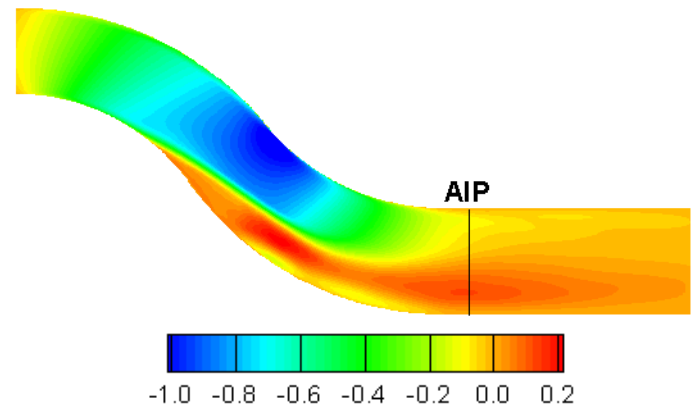

(c) $\langle v\rangle /\left\langle\bar{w}_{A I P}\right\rangle$

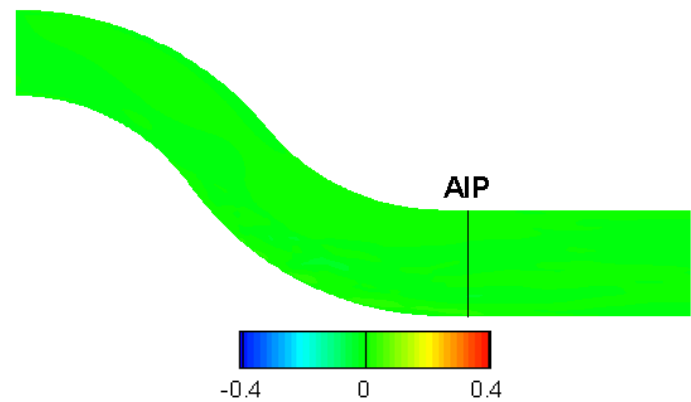

(e) $\langle u\rangle /\left\langle\bar{w}_{A I P}\right\rangle$ (b) $\sigma_{w} /\left\langle\bar{w}_{A I P}\right\rangle$

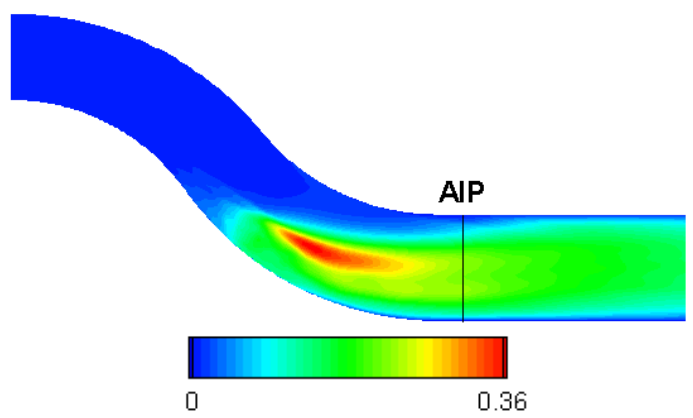

(d) $\sigma_{v} /\left\langle\bar{w}_{A I P}\right\rangle$

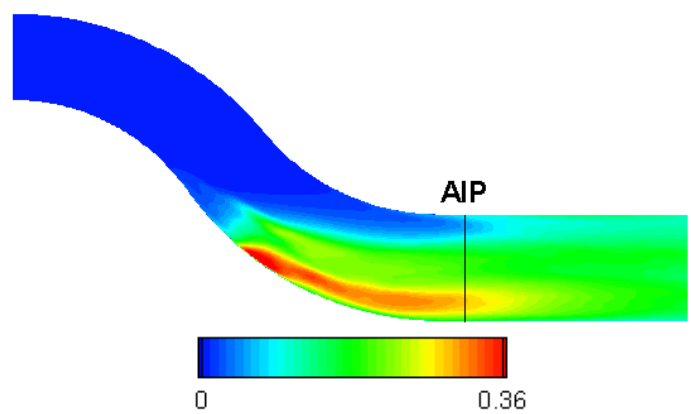

(f) $\sigma_{u} /\left\langle\bar{w}_{A I P}\right\rangle$

Fig. 10 Time-averaged and standard-deviation velocity field at the symmetry plane (DDES, $\left.M_{\text {ref }}=0.27\right)$

\section{Multi-plane Proper Orthogonal Decomposition}

PODis applied to the three-component velocity vector at the AIP and symmetry plane simultaneously. This multiplane POD permits the identification of coherent structures at the AIP and their relationship with the upstream flow field. Therefore this technique establishes a link between the upstream flow and the perturbations at the AIP. To the author's knowledge this is the first attempt to relate AIP and symmetry plane flow characteristics using POD in Sduct research. As this multi-plane POD is now based on the $\langle\overline{T K} \bar{E}\rangle$ of both the AIP and the symmetry plane, it could be expected that there will be a change in the modal distributions. However the four most-energetic modal perturbations at the AIP obtained with the multi-plane POD (Fig. 11a,b and Fig. 13a,b) are similar to those obtained previously with the POD applied only to the AIP velocity field (Fig. 7a,e,i,m).

\section{Swirl switching}

The First Switching Mode (FSM) does not show any perturbations either in the vertical or stream-wise velocity components at the symmetry plane. However, as expected, this perturbation promotes significant perturbations in the lateral-velocity field at the symmetry plane (Fig. 11c). The FSM shows a series of alternate positive and negative lateral-velocity regions along the symmetry plane, which are tilted by about $25-30^{\circ}$ relative to the stream-wise axis. 
At any cross section perpendicular to the stream-wise axis, the symmetry plane shows two regions of opposite-sign lateral-velocity, one on top of the other (Fig. 11c). This indicates the dominance of one of the two secondary flow vortices which migrate towards a more central position in the cross-section, while the other vortex is confined to the opposite wall as observed in Section III B above (Fig. 8c,d). Depending on the stream-wise position along the duct, either the clockwise or anti-clockwise vortex dominates the section (Fig. 11c). The origin of the swirl switching oscillation is located downstream of the separation region, at approximately the position of the mean-flow centreline reattachment point. When the temporal coefficient associated with the FSM changes sign, the swirl switching occurs at each section so that the opposite vortex becomes dominant. This represents the stream-wise convection of these swirling structures. Accordingly an opposite-sign dominant vortex is shed from downstream of the separation region. This perturbation shows an almost periodic behaviour at a frequency of about $\mathrm{St}=0.53$ (Fig. 11e), which is in agreement with the dominant frequency obtained with the POD applied only to the AIP velocity field (Fig. 9a).

The regions of alternating lateral velocity are associated with the stream-wise convection of the dominant swirling structure shed from downstream of the separation region. However the gap between these regions of the FSM (Fig. 11c) does not allow for a full representation of the stream-wise convection process. The Second Switching Mode (SSM) needs to be considered to complete the representation of the swirl switching mechanism. This perturbation shows the same periodic behaviour at $\mathrm{St}=0.53$ as the FSM (Fig. 11f). The spectral coherence and phase between $a_{F S M}(t)$ and $a_{S S M}(t)$ was computed, and showed that these coefficients are out-of-phase by approximately $90^{\circ}$ at $\mathrm{St}=0.53$. The perturbation of the lateral velocity field at the symmetry plane is similar to that in the FSM, but in this case the oscillating regions are interleaved between the regions of the FSM (Fig. 11d). When the contributions of the FSM and SSM are merged together, the result is a continuous downstream convection of alternate positive and negative lateral velocity structures. Therefore the multi-plane POD shows that both FSM and SSM need to be considered to fully describe the swirl switching mechanism. Half of the periodic swirl switching cycle is illustrated in Fig. 12, based on the reconstruction of the flow field with just FSM and SSM super-imposed to the mean-flow. The AIP in-plane streamlines permits the visualisation of the vortex switching. The swirl switching cycle starts with a single clockwise rotating structure which spans over the full AIP (Fig. 12b). This structure becomes progressively confined towards the bottom wall (Fig. 12e,h), until eventually another structure rotating in the opposite direction appears on top of the original structure (Fig. 12k). The new structure becomes dominant while the original vortex diminishes (Fig. 12n). The same mechanism is then repeated in the opposite direction which returns the streamlines 
pattern to the starting point (Fig. 12b). This flow mechanism occurs at a frequency of $\mathrm{St}=0.53$. The swirl switching is accompanied by a lateral oscillation of the low stream-wise velocity region (Fig. 12a,d,g,j,m). This is in agreement with the observations by Tunstall et al. [17], who suggested that the swirl switching could be caused by the lateral movement of the flow separation towards one side, relative to its mean position centred in the symmetry plane. This would allow more fluid to flow on paths of higher curvature in the opposite half of the pipe cross-section, which then results in the dominance of the associated vortex. The swirl switching at the AIP is associated with the downstream convection of the alternating lateral velocity regions along the symmetry plane, which occur as a result of the alternate shedding of opposite-sign vortices from the rear end of the separation region (Fig. 12c,f,i,l,o). Therefore the AIP perturbations highlighted by the FSM (Fig. 11a) and SSM (Fig. 11b) are promoted by the same swirl switching mechanism, which has a different impact on the AIP velocity field at the different stages during the downstream convection.

First Switching Mode (FSM)

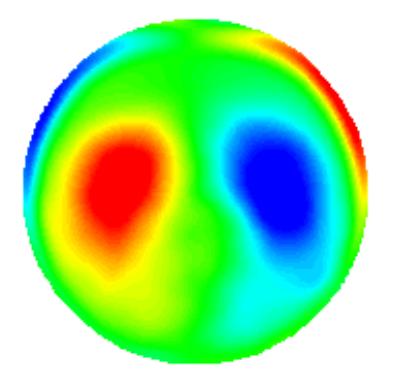

Second Switching Mode (SSM)

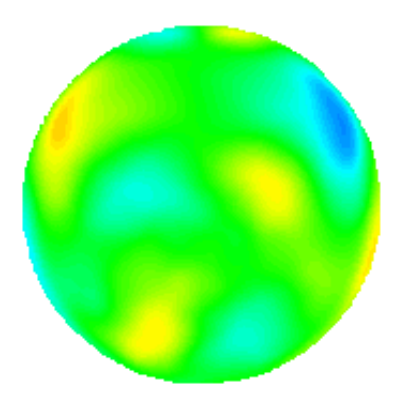

American Institute of Aeronautics and Astronautics 


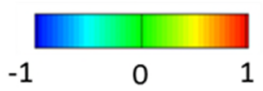

(a) $\Phi_{F S M}^{w}$ at the AIP

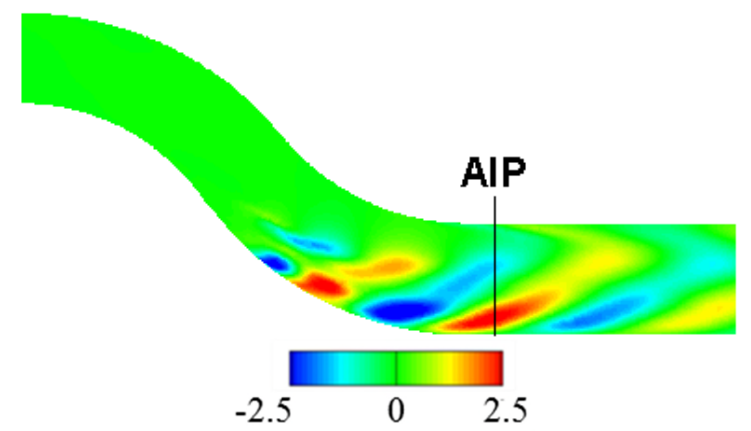

(c) $\Phi_{F S M}^{u}$ at the symmetry plane

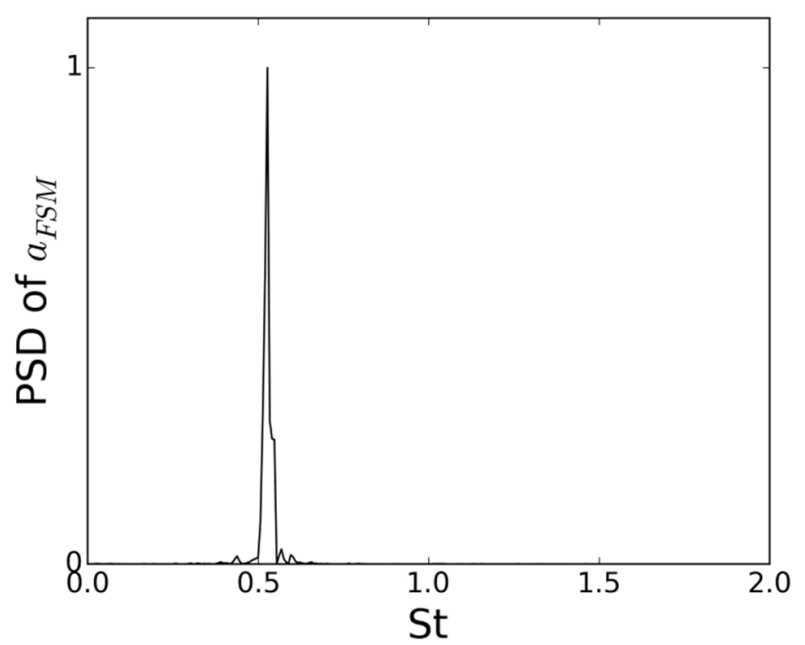

(e) Normalised PSD of $a_{F S M}(t)$

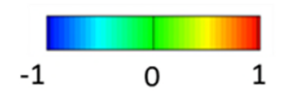

(b) $\Phi_{S S M}^{w}$ at the AIP

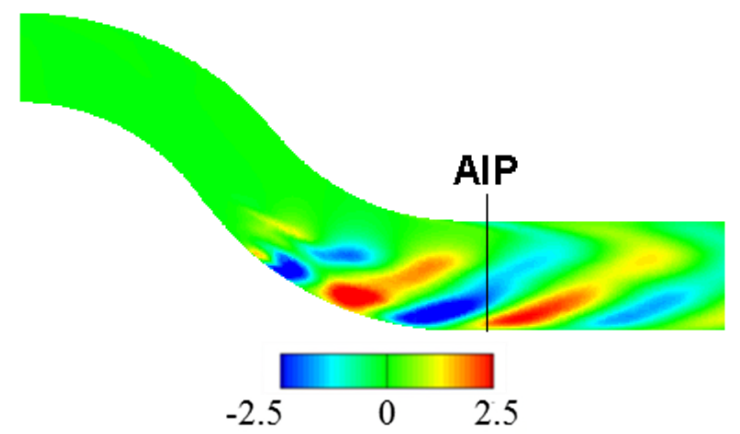

(d) $\Phi_{S S M}^{u}$ at the symmetry plane

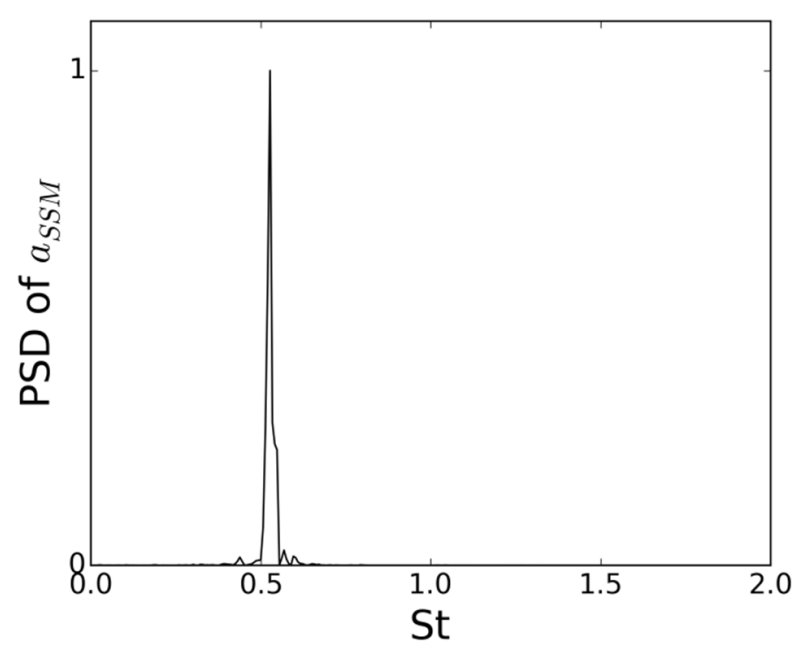

(f) Normalised PSD of $a_{S S M}(t)$

Fig. 11 FSM and SSM of the combined AIP and Symmetry plane velocity field POD (DDES, $M_{\text {ref }}=0.27$ )

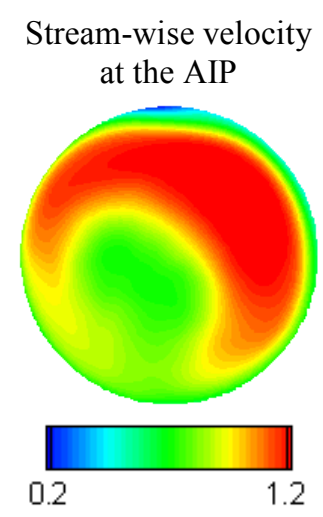

(a) $w /\left\langle\bar{w}_{A I P}\right\rangle, \mathrm{t} / \mathrm{T}=0.00$
In-plane velocity at the AIP

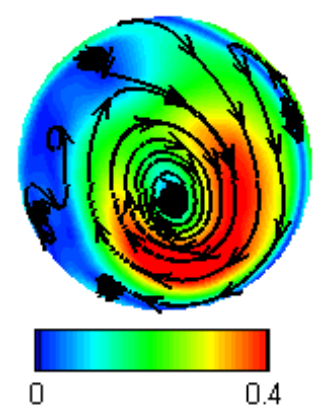

(b) $V_{i p} /\left\langle\bar{w}_{A I P}\right\rangle, \mathrm{t} / \mathrm{T}=0.00$
Lateral velocity at the symmetry plane

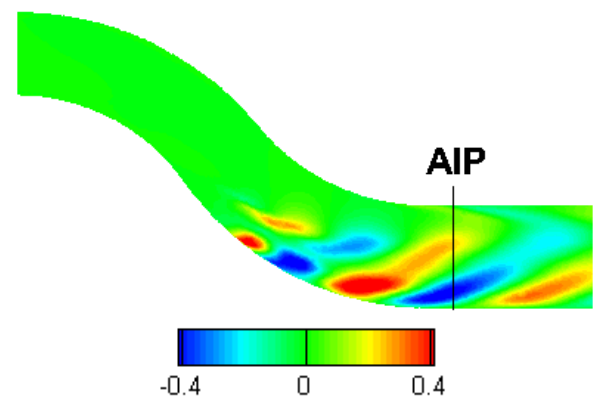

(c) $u /\left\langle\bar{w}_{A I P}\right\rangle, t / T=0.00$

American Institute of Aeronautics and Astronautics 


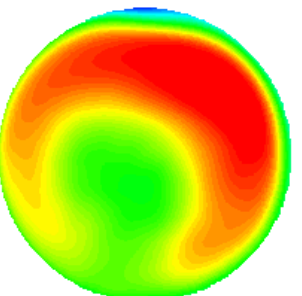

(d) $w /\left\langle\bar{w}_{A I P}\right\rangle, \mathrm{t} / \mathrm{T}=0.12$

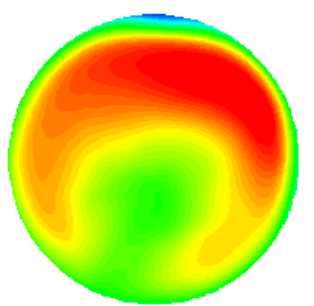

(g) $w /\left\langle\bar{w}_{A I P}\right\rangle, \mathrm{t} / \mathrm{T}=0.25$

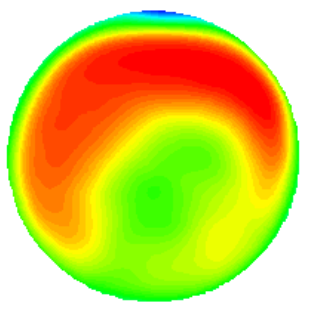

(j) $w /\left\langle\bar{w}_{A I P}\right\rangle, \mathrm{t} / \mathrm{T}=0.37$

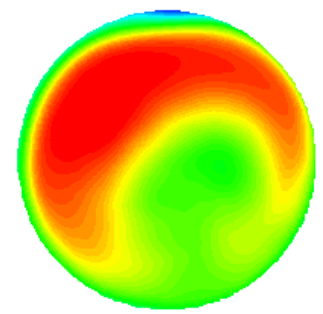

(m) $w /\left\langle\bar{w}_{A I P}\right\rangle, \mathrm{t} / \mathrm{T}=0.50$

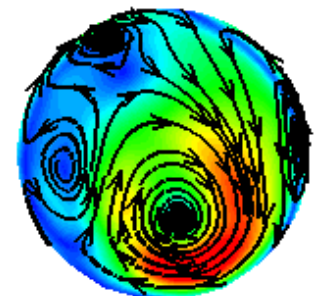

(e) $V_{i p} /\left\langle\bar{w}_{A I P}\right\rangle, \mathrm{t} / \mathrm{T}=0.12$

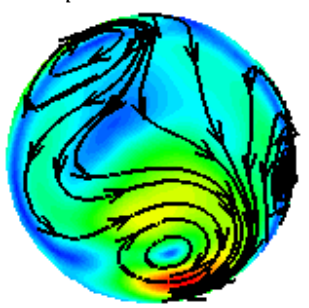

(h) $V_{i p} /\left\langle\bar{w}_{A I P}\right\rangle, \mathrm{t} / \mathrm{T}=0.25$

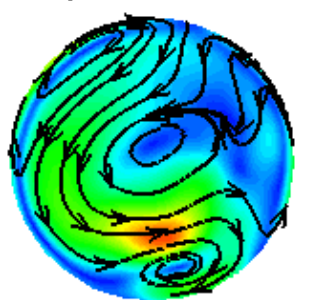

(k) $V_{i p} /\left\langle\bar{w}_{A I P}\right\rangle, \mathrm{t} / \mathrm{T}=0.37$

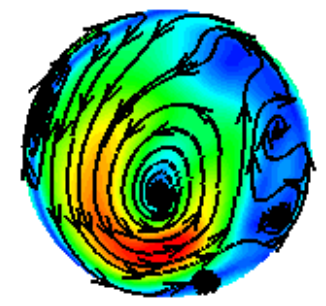

(n) $V_{i p} /\left\langle\bar{w}_{A I P}\right\rangle, \mathrm{t} / \mathrm{T}=0.50$

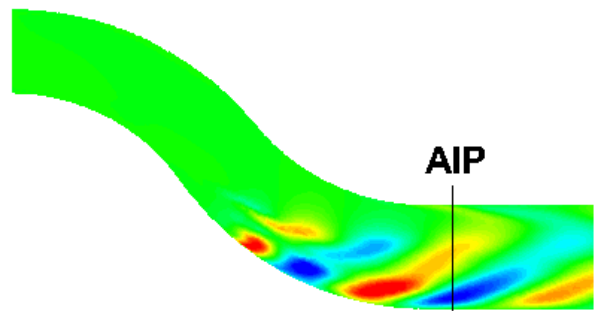

(f) $u /\left\langle\bar{w}_{A I P}\right\rangle, \mathrm{t} / \mathrm{T}=0.12$

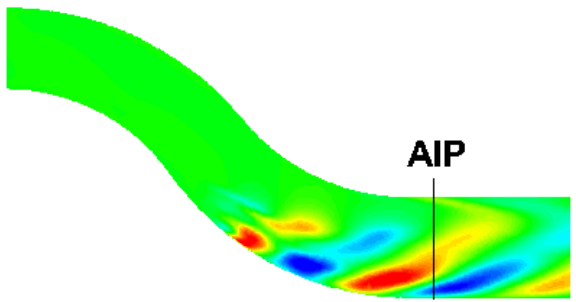

(i) $u /\left\langle\bar{w}_{A I P}\right\rangle, \mathrm{t} / \mathrm{T}=0.25$

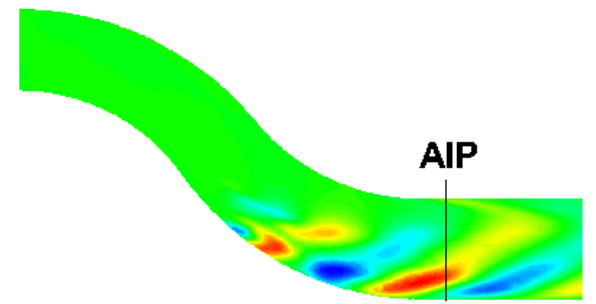

(l) $u /\left\langle\bar{w}_{A I P}\right\rangle, \mathrm{t} / \mathrm{T}=0.37$

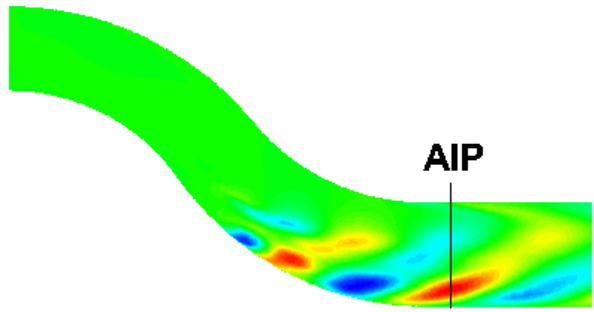

(o) $u /\left\langle\bar{w}_{A I P}\right\rangle, \mathrm{t} / \mathrm{T}=0.50$

Fig. 12 Swirl switching half-cycle reconstruction with the mean-flow, FSM and SSM (DDES, M $\left._{\text {ref }}=0.27\right)$

First Vertical Mode (FVM)

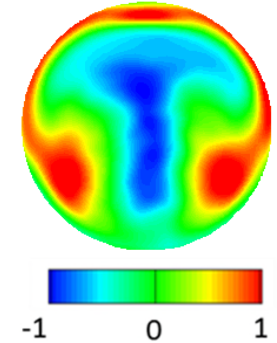

(a) $\Phi_{F V M}^{w}$ at the AIP
Second Vertical Mode (SVM)

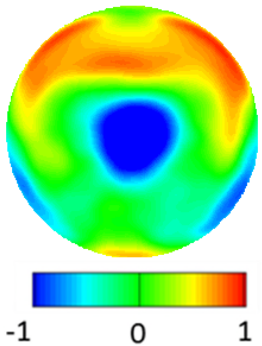

(b) $\Phi_{S V M}^{w}$ at the AIP 


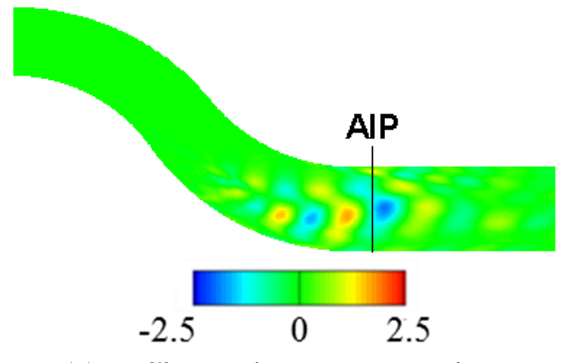

(c) $\Phi_{F V M}^{W}$ at the symmetry plane

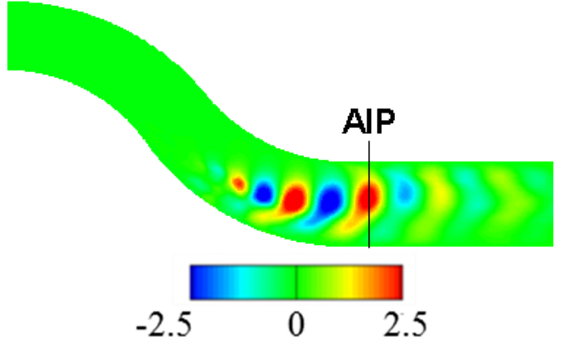

(e) $\Phi_{F V M}^{v}$ at the symmetry plane

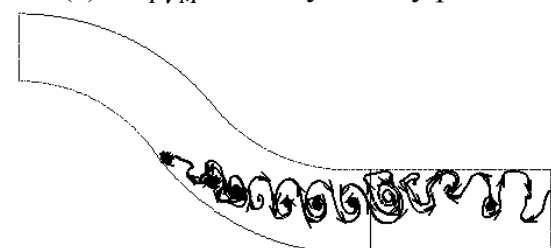

(g) $\Phi_{F V M}^{v, w}$ streamlines at the symmetry plane

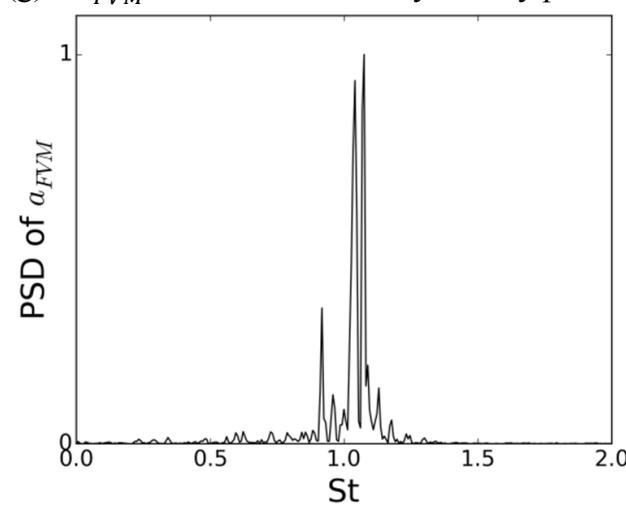

(i) Normalised PSD of $a_{F V M}(t)$

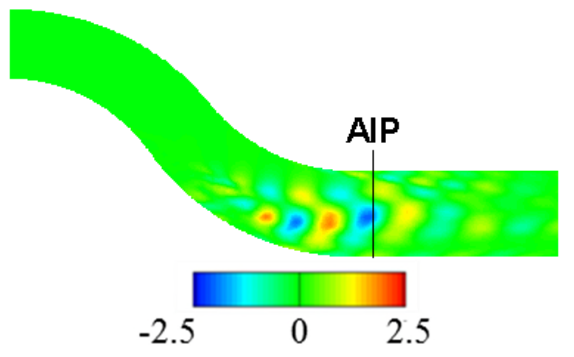

(d) $\Phi_{S V M}^{W}$ at the symmetry plane

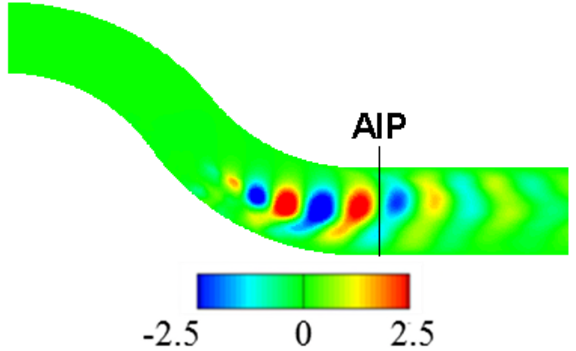

(f) $\Phi_{S V M}^{v}$ at the symmetry plane

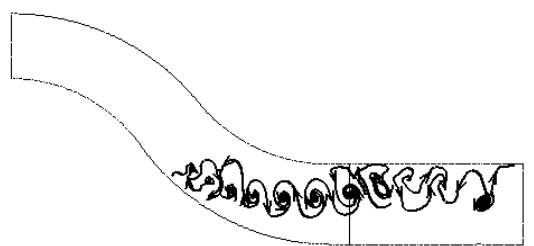

(h) $\Phi_{S V M}^{v, w}$ streamlines at the symmetry plane

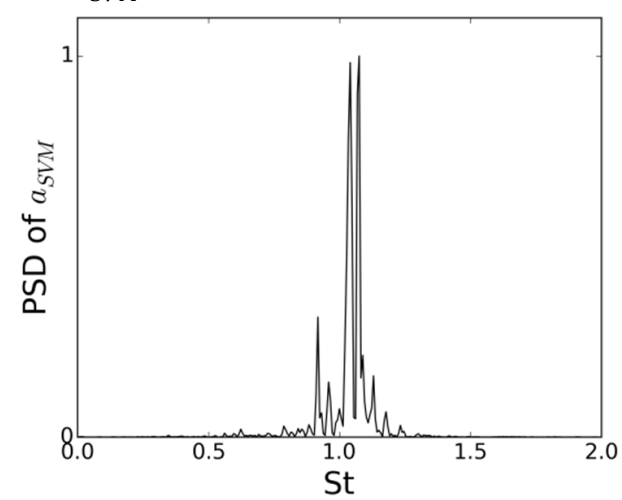

(j) Normalised PSD of $a_{S V M}(t)$

Fig. 13 FVM and SVM of the combined AIP and Symmetry plane velocity field POD (DDES, $\left.M_{\text {ref }}=0.27\right)$

\section{Shear layer oscillations}

The AIP perturbation promoted by the First Vertical Mode (FVM) of the multi-plane POD (Fig. 13a) is similar to that obtained with the POD applied just at the AIP (Fig. 7e), even though the central region of the modal shape is more spread vertically. The symmetry plane velocity field does not show any perturbation in the lateral velocity and the perturbations promoted by the FVM are only observed for the vertical and stream-wise components of the velocity 
(Fig. 13c,e). The in-plane streamlines pattern at the symmetry plane suggests that this perturbation occurs as a consequence of the shear layer unsteadiness associated with a rolled-up street of span-wise vortices which are shed from the rear-end of the separation region (Fig. 13g). The vortex shedding occurs mainly at a frequency of about $\mathrm{St}=1.06$ (Fig. 13i), which is exactly twice the value for the swirl switching mechanism (Fig. 11e,f). As occurred for the swirl switching mechanism, the downstream convection of the span-wise vortices requires the consideration of both the FVM and SVM. The AIP perturbation associated with the SVM (Fig. 13b) is similar to that obtained when only the AIP was considered (Fig. 7d). The SVM presents the same dominant frequency of St=1.06 as the FVM (Fig. 13j). The phase between $a_{F V M}(\mathrm{t})$ and $a_{S V M}(\mathrm{t})$ at $\mathrm{St}=1.06$ has been computed and is approximately $90^{\circ}$. Therefore the AIP perturbations represented by the FVM and SVM are promoted by the same flow mechanism, which is the roll-up of alternating span-wise vortices through the shear layer. The FVM and SVM also showed minor energy contributions from low frequencies for the POD applied at just the AIP velocity field (Fig. 9b,d), which are not promoted by the shear layer instability as represented by the FVM and SVM when the multi-POD approach is applied (Fig. 13i,j). These low frequency minor contributions are therefore promoted by flow features different from the shear layer unsteadiness described above.

\section{Conclusions}

The unsteady flow field in an S-duct with a centreline offset of $\mathrm{H} / \mathrm{L}=0.50$, area ratio of $\mathrm{AR}=1.52$ and length $\mathrm{L} / \mathrm{D}_{\mathrm{in}}=4.95$ has been simulated at $\mathrm{M}_{\mathrm{ref}}=0.27$ and $\mathrm{Re}_{\mathrm{D}}=7.1 \times 10^{5}$ using a DDES approach. SPIV measurements at the AIP have been used to validate the computational results. Very good agreement has been found between computational and experimental data for the mean-flow field of the three components of the velocity. The DDES simulation is also able to predict the regions of maximum flow unsteadiness even though the fluctuating levels are slightly over-predicted in general compared with the experimental results. The POD of the AIP velocity vector field reveals the same coherent structures for both DDES and SPIV data. Therefore the capability of DDES simulations to capture the main unsteady characteristics of flows within S-duct intakes has been demonstrated.

The main coherent structures of the flow are referred to as First Switching Mode, Second Switching Mode, First Vertical Mode and Second Vertical Mode. A novel approach in S-duct research based on the simultaneous POD of the velocity field at both the AIP and symmetry plane has been undertaken. This multi-plane POD technique permits the identification of the upstream origin of the AIP perturbations which determine the unsteady distortion 
characteristics of the flow delivered to the engine. This is of interest for the design of efficient flow control systems. The First and Second Switching Modes describe the swirl switching mechanism, by which alternately a dominant clockwise or counter-clockwise stream-wise vortex is shed from downstream of the separation region at a frequency of $\mathrm{St}=0.53$. The stream-wise vortices are then convected downstream and promote a swirl switching oscillation in the AIP velocity field. A period of the swirl switching oscillation has been described at the AIP. The initially dominant vortex is progressively confined to the lower sector of the AIP, and eventually a second vortex appears on top of the original swirling structure. The new vortex gradually becomes dominant until the original vortex eventually diminishes. Then the same mechanism occurs so that an opposite-rotating vortex becomes dominant, and the cycle starts again. The Switching Mode perturbation at the AIP is accompanied by a circumferential modulation of the main loss region. The First and Second Vertical Modes are associated with the unsteadiness of the centreline shear layer, which appears to be associated with the shedding of a street of span-wise vortices from the separation region. The main shedding frequency is about $\mathrm{St}=1.06$, which is twice the frequency associated with the swirl switching mechanism. The span-wise vortices are convected downstream and perturb the AIP vertical and stream-wise velocity fields. The result is a vertical modulation of the main loss region, and a fluctuation of the stream-wise velocity gradient between the high and low velocity regions at the AIP.

Overall this work demonstrates the feasibility of DDES simulations to capture the unsteady characteristics of the highly turbulent flow field in S-ducts. The most-energetic coherent structures at the AIP have been identified which determine the dynamic distortion characteristics of the flow field delivered to the engine. The upstream origin of these perturbations has been revealed as well as the fundamental frequency associated with these flow mechanisms. The identification of the source of the AIP perturbations may facilitate the design of more efficient flow control systems.

\section{Acknowledgments}

The authors would like to thank Alessandro Soli for his contribution.

\section{References}

[1] Kawai, R. T., Friedman, D. M., and Serrano, L., "Blended Wing Body (BWB) Boundary Layer Ingestion (BLI) Inlet Configuration and System Studies,” NASA Technical Report CR-2006-214534, Dec. 2006.

[2] Kim, H., and Liou, M., "Flow Simulation of N2B Hybrid Wing Body Configuration," 50th AIAA Aerospace Sciences Meeting including the New Horizons Forum and Aerospace Exposition, AIAA 2012-0838, Nashville, Tennessee, USA, Jan. 2012. 
[3] Society of Automotive Engineers, “A Methodology for Assessing Inlet Swirl Distortion,” SAE Aerospace Information Report 5686, Warrendale, PA, USA, 2007.

[4] Society of Automotive Engineers, "Inlet Total-Pressure-Distortion Considerations for Gas-Turbine Engines," SAE Aerospace Information Report 1419a, Warrendale, PA, USA, 1999.

[5] Kidman, D. S., Reagan, P. V., and Malloy, D. J., "Comparison of Inlet Compatibility Results From Subscale Wind Tunnel and Full-Scale Flight Tests of the F/A-22 Aircraft with the F119-PW-100 Engine," 17th Symposium on Air Breathing Engines, International Society of Air Breathing Engines, ISABE-2005-1169, 2005.

[6] Wenzel, L. M., and Blaha, R. J., “Analysis of Dynamic Inlet Distortion Applied to a Parallel Compressor Model,” NASA TM X-3522, May 1977.

[7] Rademakers, R. P. M., Bindl, S., and Niehuis, R., "Effects of Flow Distortions as They Occur in S-Duct Inlets on the Performance and Stability of a Jet Engine," ASME Journal of Engineering for Gas Turbines and Power, Vol. 138, No. 2, Sep. 2015, p. 022605. Doi: 10.1115/1.4031305

[8] Cousins, W., T., "History, Philosophy, Physics and Future Directions of Aircraft Propulsion System / Inlet Integration," Proceedings of ASME Turbo Expo 2004: Power for Land, Sea, and Air, Vol. 2, GT2004-54210, June 2004, pp. 305-320. Doi: $10.1115 /$ GT2004-54210

[9] Bowditch, D. N., and Coltrin, R. E., “A Survey of Inlet/Engine Distortion Compatibility,” NASA TM-83421, June 1983.

[10] Stevens, C. H., Spong, E. D., and Hammock, M. S., "F-15 Inlet/Engine Test Techniques and Distortion Methodologies Studies. Volume 1: Technical Discussion,” NASA CR-144866, June 1978.

[11] Wellborn, S. R., Reichert, B. A., and Okiishi, T. H., "Study of the Compressible Flow in a Diffusing S-Duct," AIAA Journal of Propulsion and Power, Vol. 10, No. 5, Sept.-Oct. 1994, pp. 668-675. Doi: 10.2514/3.23778

[12] Berens, T. M., Delot, A. L., Chevalier, M., and Van Muijden, J., "Numerical Simulations for High Offset Intake Diffuser Flows," 52nd AIAA Aerospace Sciences Meeting - AIAA SciTech 2014, AIAA 2014-0371, National Harbor, Maryland, USA, Jan. 2014 . Doi: 10.2514/6.2014-0371

[13] Garnier, E., "Flow Control by Pulsed Jet in a Curved S-Duct: A Spectral Analysis," AIAA Journal, Vol. 53, No. 10, Oct. 2015, pp. 2813-2827. Doi: 10.2514/1.J053422

[14] Zachos, P. K., MacManus, D. G., Gil-Prieto, D., and Chiereghin, N., "Flow Distortion Measurements in Convoluted Aero Engine Intakes,” AIAA Journal, Vol. 54, No. 9, Sep. 2016, pp. 2819-2832. Doi: 10.2514/1.J054904

[15] MacManus, D. G., Chiereghin, N., Gil-Prieto, D., and Zachos, P., "Complex Aero-Engine Intake Ducts and Dynamic Distortion," 33 ${ }^{\text {rd }}$ AIAA Applied Aerodynamics Conference, AIAA Aviation 2015, AIAA 2015-3304, Dallas, TX, 2015. Doi: $10.2514 / 6.2015-3304$ 
[16] Gil-Prieto, D., MacManus, D. G., Zachos, P., Tanguy, G., and Menzies, K., “Convoluted Intake Distortion Measurements Using Stereo Particle Image Velocimetry," 34 $4^{\text {th }}$ AIAA Applied Aerodynamics Conference, AIAA Aviation 2016, AIAA 20163560, Washington, D.C., 2016. Doi: 10.2514/6.2016-3560

[17] Tunstall, M. J., and Harvey, J. K., “On the effect of a sharp bend in a fully developed turbulent pipe-flow,” Journal of Fluid Mechanics, Vol. 34, No. 3, Dec. 1968, pp. 595-608. Doi: 10.1017/S0022112068002107

[18] Kalpakli Vester, A., Örlü, R., and Alfredsson, P. H., "POD analysis of the turbulent flow downstream a mild and sharp bend," Experiments in Fluids, Vol. 56, No. 3, Article id. 57, 15 pp., March 2015. Doi: 10.1007/s00348-015-1926-6

[19] Tanguy, G., MacManus, D. G., Zachos, P., Gil-Prieto, D., and Garnier, E., "Passive flow control study in a convoluted intake using Stereo Particle Image Velocimetry," 34 th AIAA Applied Aerodynamics Conference, AIAA Aviation 2016, AIAA 20163563, Washington, D.C., 2016. Doi: 10.2514/6.2016-3563

[20] Raffel, M., Willert, C., Wereley, S., and Kompenhans, J., Particle Image Velocimetry: A Practical Guide, $2^{\text {nd }}$ ed., SpringerVerlag, Berlin, 2007, Chap. 5, pp. 164-176. Doi: 10.1007/978-3-540-72308-0

[21] Spalart, P. R., Jou, W-H., Strelets, M., and Allmaras, S. R., "Comments on the feasibility of LES for wings, and on a hybrid RANS/LES approach," Advances in DNS/LES: Proceedings of First AFOSR International Conference on DNS/LES, Louisiana Tech University, Ruston, Louisiana, USA, Greyden Press, 4-8 Aug. 1997, pp. 137-147.

[22] Spalart, P. R., Deck, S., Shur, M. L., Squires, K. D., Strelets, M. K., and Travin, A., “A new version of detached-eddy simulation, resistant to ambiguous grid densities," Theoretical and Computational Fluid Dynamics, Vol. 20, May 2006, pp. 181-195. Doi: 10.1007/s00162-006-0015-0

[23] ANSYS, “ANSYS ICEM CFD User’s Manual,” ANSYS ICEM CFD 15.0, ANSYS, Inc., Canonsburg, PA, USA, Nov. 2013, Chap. 5 , p. 46

[24] ANSYS, “ANSYS Fluent Theory Guide,” Release 15.0, ANSYS, Inc., Canonsburg, PA, USA, Nov. 2013, Chap. 4, pp. 95-97

[25] Kostas, J., Soria, J., and Chong, M. S., “A comparison between snapshot POD analysis of PIV velocity and vorticity data," Experiments in Fluids, Vol. 38, No. 2, 2005, pp. 146-160. Doi: 10.1007/s00348-004-0873-4

[26] Berkooz, G., Holmes, P., and Lumley, J. L., "The proper orthogonal decomposition in the analysis of turbulent flows," Annual Review of Fluid Mechanics, Vol. 25, 1993, pp. 539-575. Doi: 10.1146/annurev.fl.25.010193.002543

[27] Gissen, A. N., Vukasinovic, B., and Glezer, A., "Dynamics of flow control in an emulated boundary layer-ingesting offset diffuser," Experiments in Fluids, Vol. 55:1794, Aug. 2014, pp. 1-19. Doi: 10.1007/s00348-014-1794-5

[28] Lumley, J. L., Stochastic Tools in turbulence, Dover ed., Dover Publications, Inc., New York, USA, 2007, Chap. 3, pp. 5759.

[29] Sirovich, L., "Turbulence and the dynamics of coherent structures. Part 1: Coherent structures," Quaterly of Applied Mathematics, vol. XLV, Oct. 1987, pp. 561-571. 
[30] Doane, D. P., and Seward, L. E., “Measuring Skewness : A Forgotten Statistic?,” Journal of Statistics Education, Vol. 19, No. 2, 2011, pp. 1-18.

[31] Balanda, K. P., and MacGillivray, H. L., “Kurtosis: A Critical Review,” The American Statistician, Vol. 42, No. 2, May 1988, pp. 111-119. Doi: 10.1080/00031305.1988.10475539

[32] Gordeyev, S. V., and Thomas, F. O., "Coherent structure in the turbulent planar jet. Part 2. Structural topology via POD eigenmode projection,” Journal of Fluid Mechanics, Vol. 460, 2002, pp. 349-380. Doi: 10.1017/S0022112002008364

[33] Délery, J., Three-dimensional Separated Flow Topology. Critical Points, Separation Lines and Vortical Structures, Focus series in fluid mechanics, ISTE Ltd and John Wiley \& Sons, Inc., 2013, pp. 9, 32-36. 
2016-06-30

\title{
Dynamic flow distortion investigation in an S-duct using DDES and SPIV data
}

\author{
Gil-Prieto, Daniel
}

American Institute of Aeronautics and Astronautics

Daniel Gil-Prieto, David G. MacManus, Pavlos Zachos, Geoffrey Tanguy, François Wilson, and Nicola Chiereghin. Dynamic Flow Distortion Investigation in an S-duct using DDES and SPIV data. 34th AIAA Applied Aerodynamics Conference, AIAA AVIATION Forum, 13-17 June, Washington DC, USA. Paper number AIAA 2016-3562

http://dx.doi.org/10.2514/6.2016-3562

Downloaded from Cranfield Library Services E-Repository 